\title{
Simulations of galactic winds and starbursts in galaxy clusters
}

\author{
W. Kapferer ${ }^{1}$, C. Ferrari ${ }^{1}$, W. Domainko ${ }^{1}$, M. Mair ${ }^{1}$, T. Kronberger ${ }^{1,2}$, S. Schindler ${ }^{1}$, S. Kimeswenger ${ }^{1}$, \\ E. van Kampen ${ }^{1}$, D. Breitschwerdt ${ }^{3}$, and M. Ruffert ${ }^{4}$
}

\author{
${ }^{1}$ Institut für Astrophysik, Leopold-Franzens-Universität Innsbruck, Technikerstr. 25, 6020 Innsbruck, Austria \\ e-mail: wolfgang.e.kapferer@uibk.ac.at \\ 2 Institut für Astrophysik, Universität Göttingen, Friedrich-Hund-Platz 1, 37077 Göttingen, Germany \\ 3 Institut für Astronomie, Türkenschanzstrasse 17, 1180 Wien, Austria \\ ${ }^{4}$ School of Mathematics, University of Edinburgh, Edinburgh EH9 3JZ, Scotland, UK
}

Received 3 August 2005 / Accepted 24 October 2005

\section{ABSTRACT}

We present an investigation of the metal enrichment of the intra-cluster medium (ICM) by galactic winds and merger-driven starbursts. We use combined $\mathrm{N}$-body/hydrodynamic simulations with a semi-numerical galaxy formation model. The mass loss by galactic winds is obtained by calculating transonic solutions of steady state outflows, driven by thermal, cosmic ray and MHD wave pressure. The inhomogeneities in the metal distribution caused by these processes are an ideal tool to reveal the dynamical state of a galaxy cluster. We present surface brightness, $\mathrm{X}$-ray emission weighted temperature and metal maps of our model clusters as they would be observed by X-ray telescopes like XMM-Newton. We show that X-ray weighted metal maps distinguish between pre- or post-merger galaxy clusters by comparing the metallicity distribution with the galaxy-density distribution: pre-mergers have a metallicity gap between the subclusters, post-mergers a high metallicity between subclusters. We apply our approach to two observed galaxy clusters, Abell 3528 and Abell 3921, to show whether they are pre- or post-merging systems. The survival time of the inhomogeneities in the metallicity distribution found in our simulations is up to several Gyr. We show that galactic winds and merger-driven starbursts enrich the ICM very efficiently after $z=1$ in the central ( $\sim 3 \mathrm{Mpc}$ radius) region of a galaxy cluster.

Key words. galaxies: clusters: general - galaxies: abundances - galaxies: interactions - galaxies: ISM - X-ray: galaxies: clusters

\section{Introduction}

Modern X-ray observations of galaxy clusters show clearly a non-uniform, non-spherical distribution of metals in the ICM (e.g. Schmidt et al. 2002; Furusho et al. 2003; Sanders et al. 2004; Fukazawa et al. 2004; Hayakawa et al. 2004). As heavy elements are only produced in stars the processed material must have been ejected into the intra-cluster medium (ICM) by cluster galaxies. The first suggested transfer-processes were galactic winds (De Young 1978) and ram-pressure stripping (Gunn \& Gott 1972). Other processes like kinetic mass redistribution due to galaxy-galaxy interactions (Kapferer et al. 2005; Gnedin 1998), intra-cluster supernovae (Domainko et al. 2004) or jets of AGNs are the latest suggestions for enriching the ICM. In order to distinguish between the efficiency of the enrichment processes several approaches for simulations were carried out. De Lucia et al. (2004) used combined $\mathrm{N}$-body and semi-analytical techniques to model the intergalactic and intracluster chemical enrichment due to galactic winds. Another approach are Tree + SPH simulations of galaxy clusters (Tornatore et al. 2004) including galactic winds. Gnedin (1998) did combined softened lagrangian hydrodynamic (SLH) particle-particle/particle-mesh $\left(\mathrm{P}^{3} \mathrm{M}\right)$ simulations to investigate the contribution of galaxy-interactions on the enrichment of the ICM. A comparison of the efficiency between rampressure stripping and quiet galactic winds was recently done by Schindler et al. (2005).

Multiwavelength and spectroscopic observations of galaxy clusters help us to reveal the dynamical and evolutionary state of the system (Ferrari et al. 2005; Belsole et al. 2005). If there is no spectroscopic information for cluster galaxies available, questions on substructures in galaxy clusters and their dynamical state are almost impossible to address. In this paper we show the possibilities of state of the art and planned future X-ray observations to obtain information about the dynamical state of a galaxy cluster. We model galactic winds and starbursts due to galaxy mergers as enrichment processes of the ICM. The resulting X-ray surface brightness, temperature and metal maps provide the key to understand the dynamics of merging and relaxed galaxy clusters.

\section{Simulations}

\subsection{Numerical methods}

We use different code modules to calculate each of the cluster components appropriately. Figure 1 gives a short overview of 


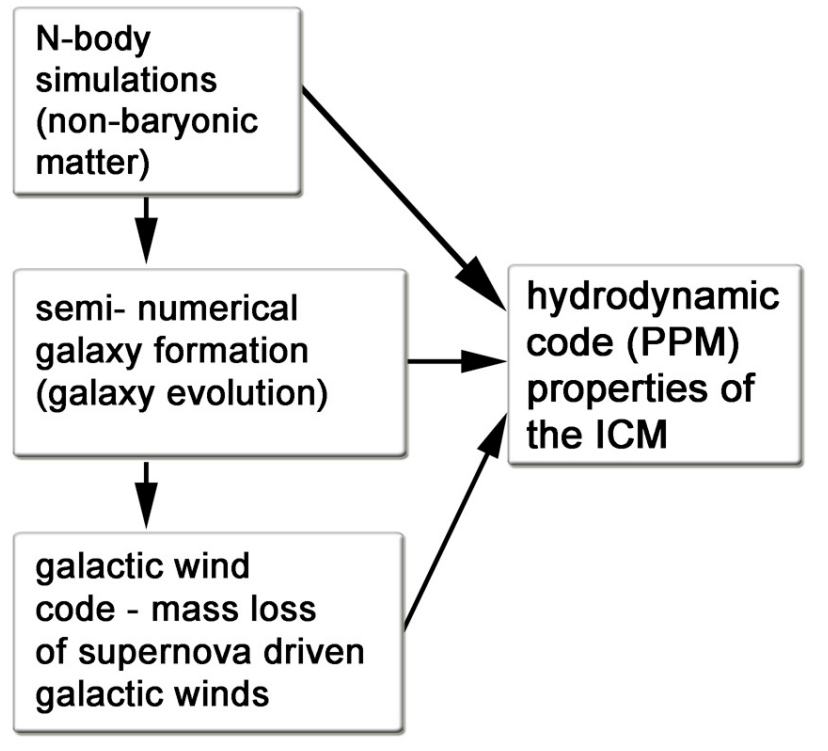

Fig. 1. Sketch of our computational scheme.

our involved techniques to simulate the several components of a galaxy cluster.

The non-baryonic component is calculated using an $N$-body tree code (Barnes \& Hutt 1986) with constrained random fields as initial conditions (Hoffman \& Ribak 1991), implemented by van de Weygaert \& Bertschinger (1996). The $N$-body tree code provides the underlying evolution of the dark matter (DM) potential for the hydrodynamic code and the orbits of the model cluster galaxies. The properties of the galaxies are calculated by an improved version of the galaxy formation code of van Kampen et al. (1999). The adopted $\Lambda$ CDM cosmology is characterized by $\Omega_{\Lambda}=0.7, \Omega_{\mathrm{m}}=0.3, \sigma_{8}=0.93$ and $h=0.7$. The mass resolution of our $N$-body simulation is in the order of $1.5 \times 10^{10} M_{\odot}$ for all three model clusters with a gravitational softening of $14 \mathrm{~h}^{-1} \mathrm{kpc}$. The improvements to the galaxy formation model of van Kampen et al. (1999) mainly concern the star formation modes, which are a mixture of bursting and quiescent star formation. Most of the recent star formation is occurring in disks, following the Schmidt law with a threshold according to the Kennicutt criterion, and most of the high-redshift star formation is resulting from merger-driven starbursts. The chemical evolution of stars and both the hot and cold components are traced, where metals are assumed to be exchanged between these components due to cooling, star formation, and feedback processes. The galaxy formation model does not eject metals from the galaxies. This is treated by the hydrodynamical code. The dark matter haloes are populated with galaxies according to the halo and galaxy merger prescriptions of the galaxy formation model, which traces their merger histories "ab initio". For each galaxy its position and velocity are known (and stored) at all times: their orbits, luminosities, colours, etc. are a product of the $N$-body simulation in combination with the assumptions of the galaxy formation model. Dark matter haloes are identified using local density percolation, as described fully in van Kampen (1997). The galaxy formation code takes into account heating of cold gas in the interstellar medium (ISM) due to supernovae and enriching the hot

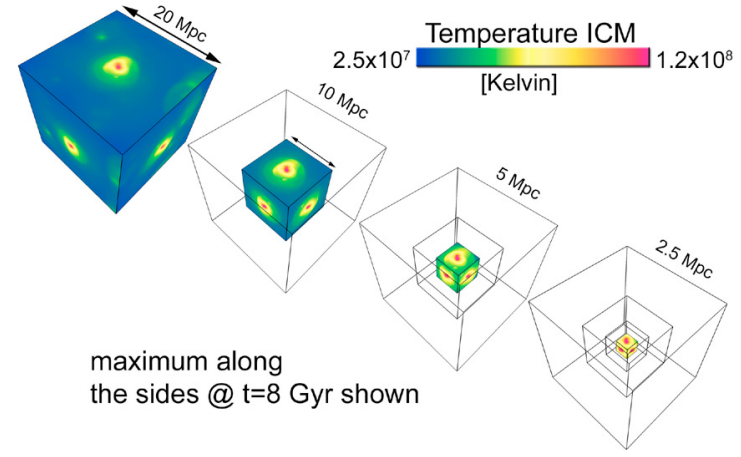

Fig. 2. The four nested grids in our mesh refinement hierarchy. The largest grid spans a volume of $(20 \mathrm{Mpc})^{3}$ the finer grids $(10 \mathrm{Mpc})^{3}$, $(5 \mathrm{Mpc})^{3}$ and $(2.5 \mathrm{Mpc})^{3}$. Each grid has a resolution of $128^{3}$ grid cells. This leads to a resolution of $(\sim 19.5 \mathrm{kpc})^{3}$ in the innermost grid.

gas in the galaxies' halos. The cooling of the supernova (SN) heated gas is modelled by radiative cooling with line emission. Besides the process of radiative cooling, the hot halo gas can be reheated up to the virial temperature by DM halo merging. This leads to a two-phase gas model for each galaxy. A hot gas phase populating the halos and a cold gas phase populating the disks of the galaxies. The whole gas is gravitationally bound to the potential field of the galaxies. Due to stellar feedback the hot gas is chemically enriched as the systems evolve, see Sect. 2.3 and van Kampen et al. (1999) for details. The fraction of mass residing in the hot and cold phases of gas depends on the $\mathrm{SN}$ rates and the energetics, i.e. masses and velocities, of the DM halo mergers.

For the treatment of the ICM we use a hydrodynamic code with shock capturing scheme (PPM, Collela \& Woodward 1984), with a fixed mesh refinement scheme (Ruffert 1992) on four levels and radiative cooling. In Fig. 2 the scheme of the fixed mesh refinement is shown.

The grids are fixed at the centre of the simulated galaxy cluster and the largest grid covers a volume of $(20 \mathrm{Mpc})^{3}$. Each finer grid covers $\frac{1}{8}$ of the next larger grid. With a resolution of $128^{3}$ grid cells in each grid we obtain a resolution of $(\sim 19.5 \mathrm{kpc})^{3}$ for each cell on the finest grid. The interaction of the $N$-body tree code with the hydrodynamic code is done as illustrated in Fig. 3.

As the $N$-body tree code, the semi-numerical galaxy formation and evolution and the mass-loss rates due to galactic winds are calculated from the beginning, the hydrodynamics covers the redshift interval from $z=1$ to $z=0$, i.e. $\sim 57 \%$ of the whole simulation time. This setup allows us to address questions like:

- what is (are) the origin(s) of the inhomogeneities found in the X-ray metallicity maps?

- how long can these inhomogeneities survive in the hot ambient ICM?

- what can we learn about the dynamical state of a galaxy cluster from X-ray observations? 
$z=20$ $z=1$

$\mathbf{z}=0$

\section{N-Body tree code}

semi-numerical galaxy formation

mass loss (galactic winds)

Initial conditions

hydrostatic equilibirium

hydrodynamic code

\section{$t=0.18$}

$\mathrm{t}=5.87 \quad[\mathrm{Gyr}]$

$\mathrm{t}=13.87$

Fig. 3. The chronology of our simulation set-up. While the $N$-body code, the galaxy formation code and the galactic winds code trace the whole evolution from the beginning the hydrodynamic covers $\sim 57 \%$ of the simulation time.

\subsection{Calculation of the mass loss due to galactic winds}

The mass loss rate due to thermally, cosmic ray and MHD wave pressure driven galactic winds is calculated for each model galaxy with a code developed by Breitschwerdt et al. (1991). For a given model galaxy the algorithm calculates the mass-loss rate and wind properties as well as the velocity of the ejected matter as a function of distance to the galaxy or the pressure flow. As input for the wind code, galaxy parameters like halo mass, disk mass, spin parameter, scale length of the components, temperature and density distribution of the ISM, as well as the stellar density distribution are required. In order to save computing time we performed parameter studies. We did more than 10.000 calculations varying the halo mass, disk mass and spin parameter to simulate a wide spectrum of different spiral galaxies. The winds were then calculated as suggested in Breitschwerdt et al. (1991). The results are summarised in a look up table. In Fig. 4 an example for a given set of galaxies is shown. All galaxies have the same halo mass of $3 \times 10^{11} M_{\odot}$. The $x$ - and $y$-axis indicate different disk masses and spin parameters $\lambda$. The combination of disk mass and spin parameter results then in a certain geometry (disk scale length) and potential (Mo et al. 1998), which then is taken as input for the galactic wind code of Breitschwerdt et al. (1991). If for a given halo mass the fraction of disk mass and the spin parameter increases, the size of the disk gets larger and therefore supernovae can explode at larger radii. The gas disk as well as the star distribution extends into the outer parts of a galaxy, where the potential is more shallow than in the inner parts. Supernovae exploding in that region can more easily accelerate the surrounding gas to the escape velocity of the system at a given galactic radius.

It is important to point out, that the mass, calculated with the above mentioned approach, is no longer gravitationally bound to the system. The mass is accelerated up to the escape speed and will leave the hot halo of the underlying galaxy. Apart from the mass loss due to galactic winds another solution is in principle possible, the so called "breeze solution".

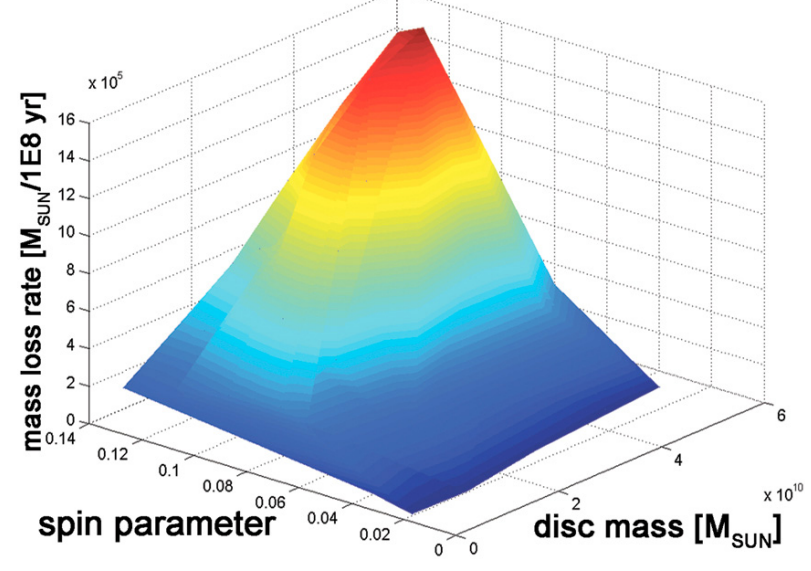

Fig. 4. The mass loss rate $\left[M_{\odot} / 1 \times 10^{8} \mathrm{yr}\right]$ for simulated galaxies as a function of disk mass and spin parameter $(\lambda)$. The halo mass for all galaxies is fixed to $3 \times 10^{11} M_{\odot}$. It can clearly be seen that higher disk masses and spin parameter result in a higher mass loss rate. This is due to the fact that higher spin parameters cause a larger disk scale length and therefore a larger disk.

In this picture gas will be accelerated to leave the galactic disk, and become then part of the hot halo gas. In this case the gas can cool radiatively and sink then towards the disk. In our hydrodynamic simulation only the ICM is modelled (several $10^{7}<T_{I C M}<$ several $10^{8}[\mathrm{~K}]$ ), therefore hot gas in the halos of galaxies is taken into account, as it is not part of the ICM. This means that the mass expelled by supersonic galactic winds does not belong to the hot halo component of the galaxies anymore and is therefore part of the ICM, which we treat in our hydrodynamic simulation.

\subsection{Calculation of the mass loss due to starbursts}

The mass loss rates due to galaxy merger induced starbursts are estimated following Heckmann et al. (2000). They investigated 32 far-IR-bright starburst galaxies and conclude that for galactic superwinds (mass loss rate $10-100 M_{\odot}$ per year) the mass outflows are comparable to the star formation rates (SFRs) of the underlying system. The SFRs in merging galaxies in our semi-numerical galaxy model are enhanced up to several $100 M_{\odot} / \mathrm{yr}$, depending on the relative velocities, masses and the gas content of the interacting galaxies during several 10 million years. In addition, Heckmann (2003) finds that in none of the cases radiative cooling of the coronal gas is sufficient to quench the outflow. This heuristic approach gives us the mass loss for a starbursting galaxy, which we add to the hydrodynamic simulation at the place of the starburst. The material is then spread over volumes corresponding to a typical travel time of $10^{7}$ years (typical time step). The metals are mixed with the ICM already present. The metallicity of the ejecta is taken from the semi-numerical galaxy modelling, which calculates the metals in a hot and cold gas reservoir through

$\dot{m}_{\mathrm{c}}=Z_{\mathrm{h}} \dot{M}_{\mathrm{cool}}-Z_{\mathrm{c}}(1+\beta-R) \Psi_{\mathrm{SF}}+y \Psi_{\mathrm{SF}}$

$\dot{m}_{\mathrm{h}}=Z_{\mathrm{h}} \dot{M}_{\text {cool }}+Z_{\mathrm{c}} \beta \Psi_{\mathrm{SF}}+Z_{\text {Prim }} \dot{M}_{\text {new }}$, 
Table 1. Properties of our model galaxy clusters.

\begin{tabular}{|c|c|c|}
\hline $\begin{array}{l}\text { Model } \\
\text { cluster }\end{array}$ & $\begin{array}{l}\text { Total mass inside } \\
\text { a radius of } 3 \mathrm{Mpc} \\
@ z=0\left[10^{14} M_{\odot}\right]\end{array}$ & $\begin{array}{c}\text { \# of galaxies } \\
\text { in the inner }(5 \mathrm{Mpc})^{3} \\
\text { volume }(\text { grid } 3)\end{array}$ \\
\hline $\mathrm{A}$ & 13.0 & 830 \\
\hline B & 6.8 & 489 \\
\hline C & 7.8 & 585 \\
\hline
\end{tabular}
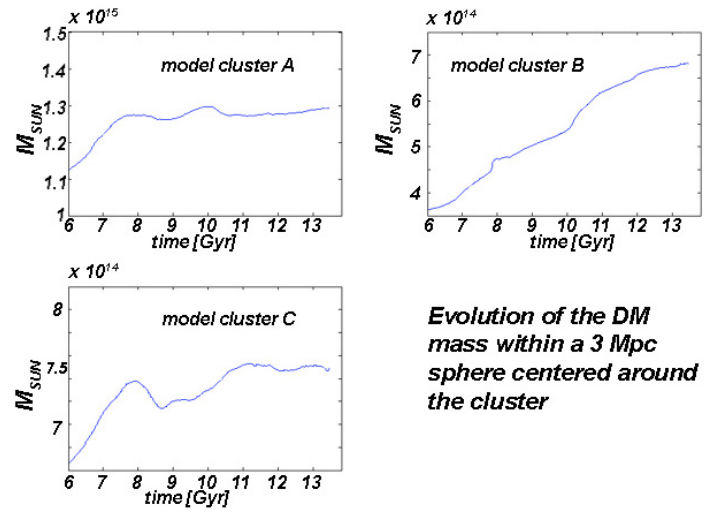

Evolution of the DM mass within a 3 Mpc sphere centered around the cluster

Fig. 5. From the mass accretion rates it is visible that model cluster $B$ is a strong merging system, model cluster $\mathrm{C}$ has less merging events and model cluster A is a relaxed, massive system. The Evolution is shown for a $3 \mathrm{Mpc}$ physical units sphere centered around the cluster, since $z=1$.

where $Z_{\mathrm{c}}=m_{\mathrm{c}} / M_{\mathrm{c}}, Z_{\mathrm{h}}=m_{\mathrm{c}} / M_{\mathrm{h}}, \dot{M}_{\text {new }}$ is the accretion of new baryons by the halo, $\Psi_{\mathrm{SF}}$ is the star formation rate, $Z_{\text {prim }}$ the primordial metallicity and $\beta$ the fraction of supernovae of a stellar population. The starbursts are modelled such, that they eject matter with the metallicity present in the cold gas reservoir. This results in a lower limit in metallicity of the starburst ejecta.

\section{The properties of our model galaxy clusters}

Three different model galaxy clusters have been simulated, hereafter cluster $\mathrm{A}, \mathrm{B}$ and $\mathrm{C}$. We simulate a non-merging galaxy cluster, a cluster with a single subcluster merger and a less massive galaxy cluster undergoing several mergers of subclusters. In Table 1 the properties of the model clusters A, B and $\mathrm{C}$ are listed.

In Fig. 5 the evolution of the DM mass within a $3 \mathrm{Mpc}$ sphere centered around the cluster is shown. The number of galaxies in our model clusters as a function of time in the simulation volume of grid 3 (5 Mpc on the side) is shown in Fig. 6.

As model cluster B is the cluster with several major mergers, the number of galaxies in grid 3 ( $5 \mathrm{Mpc}$ per side) has a stronger increase (increase $=$ new galaxies are formed + galaxies move into the volume) with time than in the cases of the non-merging model cluster $\mathrm{A}$, and less merging system $\mathrm{C}$, which have an almost constant number of galaxies in the same volume. The average mass ratio of the subclusters and mainclusters in our simulations is around 1:2 in model cluster B, and 1:4 in model cluster $C$. In model cluster $A$, a very low mass subcluster falls towards the cluster centre almost at the

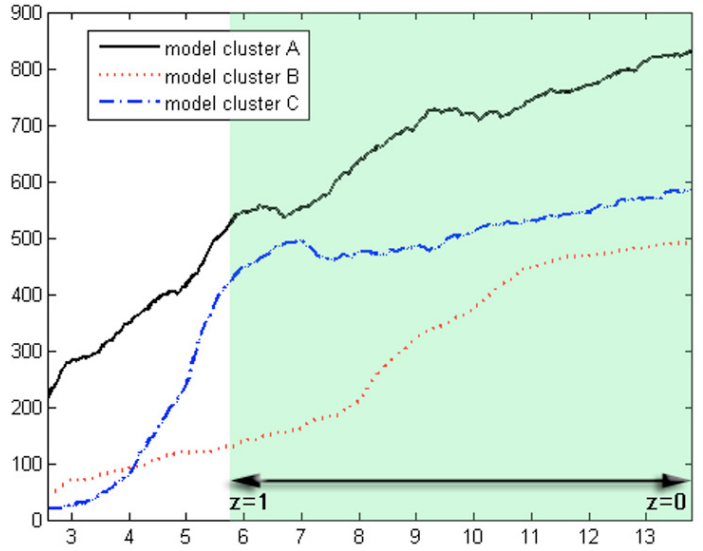

Fig. 6. Time evolution of the number of galaxies in the simulation volume grid 3 ( $5 \mathrm{Mpc}$ on a side) since $z=4$. The blue range highlights the redshift interval $z=1$ to $z=0$.

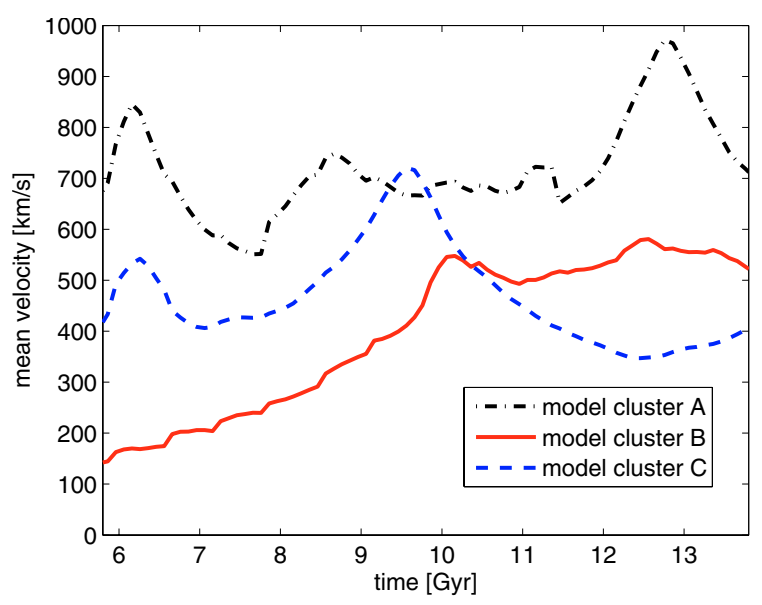

Fig. 7. Time evolution of the mean velocity of all galaxies in a central cube with $5 \mathrm{Mpc}$ on the side for model cluster A, B and C. The velocities are given starting from $z=1$ (blue range in Fig. 6).

end of the simulation. Note that the pre-merger clusters have a lot of substructure. The mean velocities of the galaxies as a function of time are shown in Fig. 7. Model cluster A has a approximately constant mean velocity over a long period and a little merger event during $1.5 \mathrm{Gyr}$ at the end of the simulation. Model cluster $\mathrm{C}$ has two major merger events, one at the beginning and one in the middle of the hydrodynamic simulation; see the two humps in the corresponding line in Fig. 7. The less massive model cluster $\mathrm{B}$ is forming during the first half of the hydrodynamic simulation by mergers of several small substructures and then in the second half there are two major mergers, at 10 and 12.5 Gyr.

In our model clusters the mass loss rate due to both quiet galactic winds and starbursts are calculated. The ratio of matter ejected by all starburst events in comparison to the galactic winds in the time interval $z=1$ to $z=0$ is $1.3 \%$ for model cluster A, $20 \%$ for model cluster B and $7.3 \%$ for model cluster $\mathrm{C}$ in a volume of $(5 \mathrm{Mpc})^{3}$ centred on the cluster. As model cluster B shows the highest number of merging events, it has the highest fraction of starburst events in the $(5 \mathrm{Mpc})^{3}$ 


\section{X-ray surface brightness} (relative logarithmic scale)

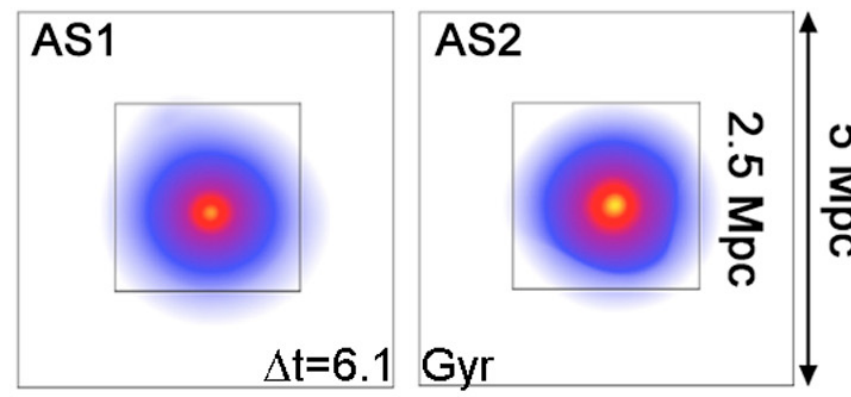

4.7

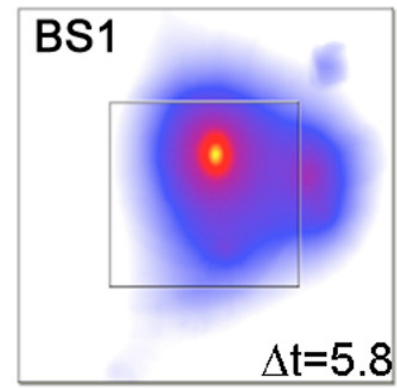

3.2

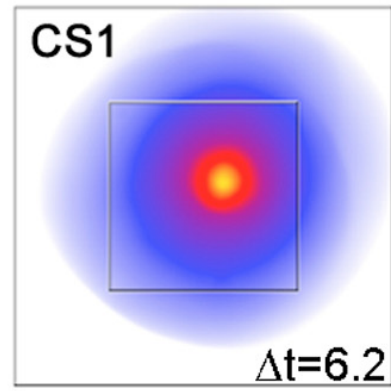

3.5

\section{4}

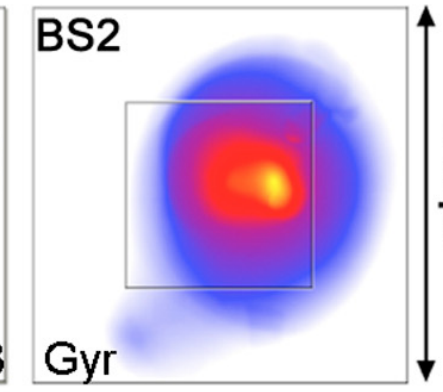

4.6

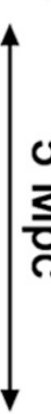

X-ray weighted temperature (linear scale)

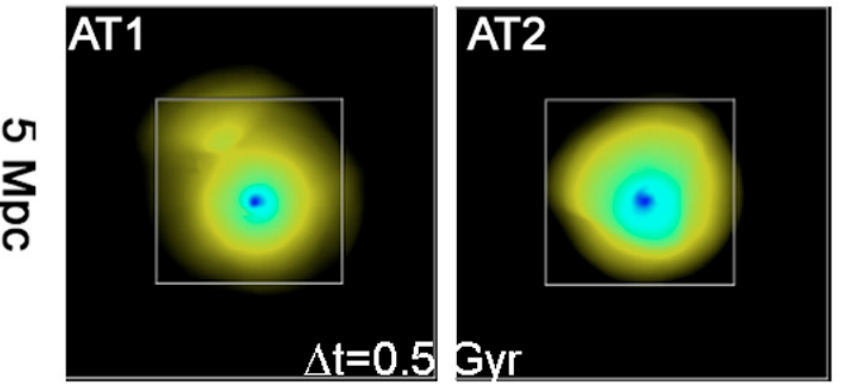

5.3
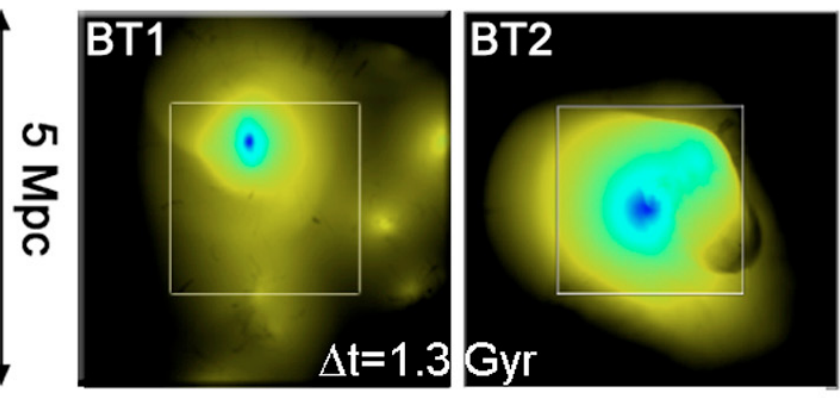

3.8

$13\left[10^{7} \mathrm{~K}\right]$

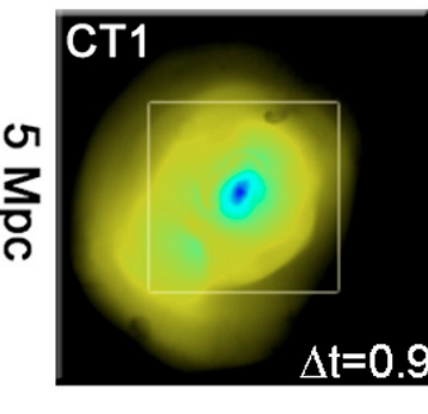

5.5

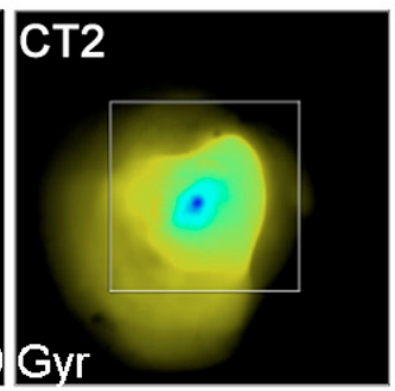

$11\left[10^{7} \mathrm{~K}\right]$

Fig. 8. X-ray surface brightness maps and X-ray weighted temperature maps for all model clusters. In the upper panel model cluster A, in the middle panel model cluster B and in the lower panel model cluster C is shown. The name for each map indicates the model cluster (A, B or C), surface brightness or temperature map $(S, T)$ and two particular timesteps 1 or 2 . The timesteps are chosen to show the clusters before and after a merger event. The X-ray surface brightness and temperature maps correspond not to the same timesteps, the main criterion for choosing them is the visibility of substructures. The range of the surface brightness maps is about 2.5 orders of magnitudes. See the text for more details.

simulation volume. This is caused by many galaxy groups moving towards the cluster centre as the cluster evolves.

\section{Results}

\subsection{X-ray maps}

We extract X-ray surface brightness, X-ray emission weighted temperature and metal maps from our simulations for comparison with observations. The images in Figs. 8 and 9 have a resolution of 256 pixels on each side (one pixel represents a region of $(19.5 \mathrm{kpc})^{2}$ and represent the projection of a volume of $(5 \mathrm{Mpc})^{3}$ on the sky).

\subsubsection{X-ray surface brightness maps}

Figure 8 shows all model clusters A, B and C at different evolutionary states. Each panel on the left hand side shows two $\mathrm{X}$-ray surface brightness maps (named S1 and S2), reproducing the X-ray emission at two different particular states in their evolution. The first letter of the name indicates the model cluster A, B or C. The maps AS1 and AS2 (upper panel) in this series show model cluster A, the most massive one with an infall of a low mass structure happening almost at the end of the simulation. In AS1 there is a small area with enhanced emission in the innermost part $(2.5 \mathrm{Mpc})^{2}$ faintly visible at the top. This feature belongs to a small subcluster. At the end of the simulation the whole cluster has become a relaxed system. 

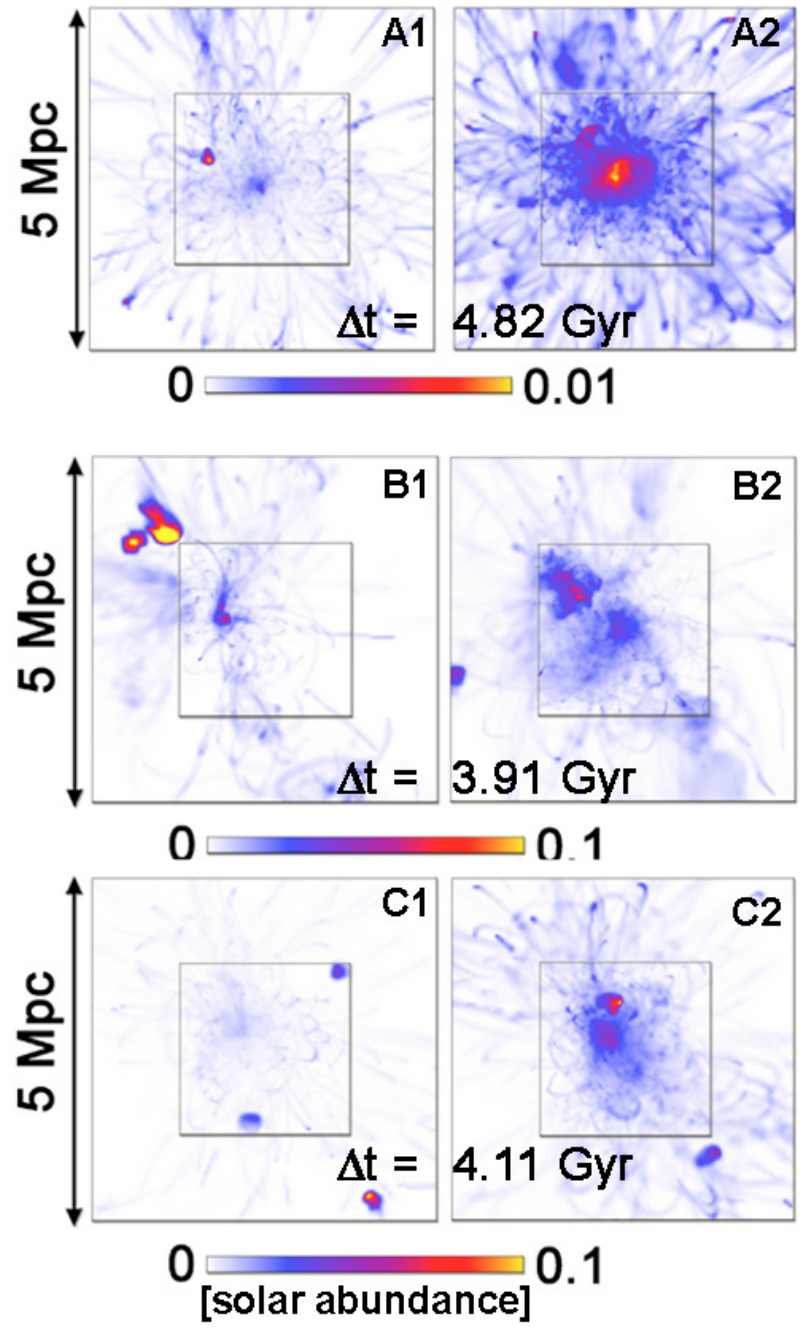

Fig. 9. X-ray metallicity maps for all model clusters. The names of the images reflect the model cluster (A, B or C) shown at 2 different timesteps. See the text for more details. Between A1 and A2 there are 4.82 Gyr, between B1 and B2 are 3.91 Gyr and between $\mathrm{C} 1$ and $\mathrm{C} 2$ 4.11 Gyr of evolution.

The maps BS1 and BS2 demonstrate the different dynamical states of model cluster B. At $z=1$ (map BS1) there are two subclusters visible, besides the main cluster. In addition there is enhanced X-ray emission due to matter ejected by a major starburst in the upper right corner. The overdensities in the surroundings of the starbursting galaxy cause the conspicuous behaviour in the X-ray surface brightness map. Map BS2 shows the state when the two substructures have already merged with the main cluster. The ejected matter due to the starburst, caused by the galaxy merger, has moved to the vicinity of the centre and is visible as a second small local maximum of X-ray emission. The elongated shape in the X-ray surface brightness map due to the merger is clearly visible. The cluster is in a post merger state.

The X-ray surface brightness map for model cluster $\mathrm{C}$ is shown in maps CS1 and CS2. The first map CS1 shows the cluster at the beginning of the hydrodynamic simulation. As the cluster undergoes one major merger event at $z=0.5$, the system does not evolve to a relaxed galaxy cluster within simulation time. In map CS2 the X-ray surface brightness map at $z=0$ is shown. The elongated structure, caused by the merger, can clearly be seen.

$\mathrm{X}$-ray surface brightness maps are able to show major merger events in galaxy clusters. X-ray observations are often limited by the number of photons arriving from the observed object and of course the intrinsic noise. Therefore it is important to mention that the maps that are shown here represent an ideal case of infinitely long observations.

\subsubsection{X-ray temperature maps}

In Fig. 8 the right images (named T1 or T2) show the X-ray weighted temperature maps of all model clusters at the same time as the same time as the left panels. Again a volume of $(5 \mathrm{Mpc})^{3}$ is represented by the maps. The two maps AT1 and AT2 show model cluster A before a small merging event and during the phase of merging. Map AT1 shows the cluster at the same time as AS1. In the X-ray temperature map the subcluster is more pronounced and the map shows in addition more structures in the centre of the main cluster, caused by several subgroups of galaxies falling in. Map AT2 shows the cluster during the merging phase $500 \mathrm{Myr}$ after map AT1. The subcluster has fallen into the main cluster and caused perturbations in the ICM, which can be seen in the aspherical shape of the cluster's temperature distribution.

Model cluster B undergoes several merging events of subclusters which can be seen in the map BT1. Besides the subclusters, map BT1 shows small, colder regions (black knots) in the ICM, which were caused by cold matter ejected by starbursts. They can be seen in the outskirts of the main cluster. In map BT2 the cluster is shown after $1.3 \mathrm{Gyr}$ of evolution. The subclusters have merged with the main cluster and strong shocks in the ICM emerge, which are visible near the upper border of the indicated $(2.5 \mathrm{Mpc})^{2}$ region. In the right upper corner of the $(2.5 \mathrm{Mpc})^{2}$ cube a cold feature is visible. This feature belongs to ejected matter by a merger-driven starburst. The ejected matter has several $10^{6} \mathrm{~K}$ and is therefore visible as cold inclusions in the ICM. The temperature of the ICM in the region of the starburst is calculated as a mixture of hot ICM with a given density and the ejecta of the starburst, which are assumed to have temperatures in the order of several $10^{6} \mathrm{~K}$. As starbursts can transport a major amount of matter into the ICM, the density increases and therefore the regions are visible in X-ray maps, see Fig. 8 - BS1.

The last model cluster $\mathrm{C}$ has a major merger event, as is clearly visible in the X-ray weighted temperature map. Map CT1 shows the subcluster and in addition cold material on the opposite side of the main cluster, which belongs to ejected matter from a starburst. In the centre of the main cluster there are several outward moving shocks showing up as hotter shell-like regions, which were caused by several very small subcluster mergers. In map CT2 the subcluster has merged with the main cluster (900 Myr later).

$\mathrm{X}$-ray weighted temperature maps are better suited to investigate the dynamical state of a galaxy cluster than the X-ray surface brightness maps. If enough photons are available in 
the X-ray observations, combination of both gives the best tool for investigating galaxy clusters in X-rays. The observed X-ray temperature maps of galaxy cluster have a limited spatial resolution, because the numbers of photons in real observations is limited.

\subsubsection{X-ray metal maps}

X-ray weighted metal maps show the strength of enrichment processes and the spatial spreading of metals. As we focus in this paper on the enrichment of the ICM by galactic winds and starbursts, the metal maps give a direct measure of their efficiency.

In Fig. 9, upper panels, two metal maps for model cluster A (A1, A2) are shown. The main striking features, which are visible, are stripes of enriched material pointing radially toward the cluster centre. These stripes belong to enriched matter ejected continuously by quiet galactic winds as the galaxies move through the ICM. As we calculate the amount of ejected matter by SN-driven galactic winds, the enriched gas is treated in the same hydrodynamic way as the ICM. In the outskirts of the galaxy cluster the stripes can survive several Gyr because the density and strength of perturbations of the ICM in enriched regions is very low. In Sect. 4.5 a more detailed analysis of the survival timescales will be given. A1 and A2 are separated by 4.82 Gyr of evolution. In A1 there is a small highly enriched region visible, which belongs to matter ejected by a mergerdriven starburst. As model cluster A is a very massive galaxy cluster (see Table 1) the pressure of the ICM on the galaxies is quite high in the central region of the cluster. Suppression of winds occurs, because enhanced $p d V$-work against the increasing ambient pressure can stall the expansion of the outflow. In our model this translates into a combined pressure of gas, cosmic rays and MHD waves, which is lower at the sonic point than the external ICM pressure. In addition to the quiescent winds, calculated in Schindler et al. (2005), we take into account starbursts caused by galaxy-galaxy interactions in this simulation. These interactions enhance the SFRs of the systems very strongly over short times (several 10 to $100 \mathrm{Myr}$, Kapferer et al. 2005) and therefore lead to short, strong outflows of matter. In A2 the highly enriched central region can be seen. The overall metallicity is very low in model cluster A, because of the suppression of quiet galactic winds by ICM pressure onto the galaxies in the central part $\left(\sim(1 \mathrm{Mpc})^{3}\right)$. Another interesting feature is the second peak in the metal map near the cluster centre (near upper-left inner corner) in the map A2 in Fig. 9, caused by the strong starburst from above. The material has not been mixed with the metals at the centre of the main cluster. It has been mixed with gas in a volume of several tens of kpc. As shown in the previous maps, model cluster A has a merger event and the induced perturbations in the ICM cause the spatial shift of this enriched region to the upper left corner of the innermost grid.

In Fig. 9 B1 and B2, the X-ray metallicity maps of model cluster B are shown. The left map shows the metallicity distribution after $4 \mathrm{Gyr}$ of evolution. Again two highly enriched regions in the outskirts of the cluster are visible. They belong to merger-driven starburst ejecta. Note that the relative velocities of galaxies, which fall in groups toward the galaxy cluster centre are significantly lower (several hundred $\mathrm{km} \mathrm{s}^{-1}$ ) than the relative velocities of the galaxies interacting at the centre of the cluster potential (thousands of $\mathrm{km} \mathrm{s}^{-1}$ ). As a consequence, the interaction times of galaxies in subgroups falling into the cluster potential are longer and merger events do have larger timescales to introduce perturbations into the inter-stellar medium, which cause the higher SFRs. Therefore starbursts due to galaxy-galaxy interactions are more effective in subgroups, falling into the cluster potential, than in the central parts of the galaxy cluster. In the metal B2 map the ICM, enriched by the starburst, has efficiently mixed with the surrounding ICM and moved towards the centre of mass, i.e. into denser regions. As a consequence the average metallicities are lower than in the left map (B1), where the ICM was not so dense in the region, where the starburst happened. Model cluster B is the least massive model cluster in our sample and does not suppress galactic winds in the central region. Although the cluster has fewer galaxies, galactic winds and starbursts can enrich the ICM an order of magnitude more efficiently than in model cluster A, the most massive cluster in our sample. This can be explained in terms of the ICM mass, which has to be enriched. A high gas mass exerts a higher pressure of the ICM onto the galaxies, especially in the central $(1 \mathrm{Mpc})^{3}$ region. Although more massive galaxy clusters have more galaxies, the higher pressure of the ICM suppresses the galactic winds of many systems in the central region. In addition, more ICM has to be enriched than in the case of a less massive cluster, which leads to lower metal abundances in our model cluster A.

In Fig. $9 \mathrm{C} 1$ and $\mathrm{C} 2$, the metal maps for the intermediate mass model cluster $\mathrm{C}$ are shown. In principle they follow the same trend as in the case of model cluster B. Again the left map shows highly enriched regions due to galaxy-merger driven starburst ejecta. As in all the other metal maps, the enriched trajectories of the galaxies can be seen in the outskirts of the galaxy cluster. These stripes can survive several Gyr, depending on the properties and the motion of the surrounding ICM. In the central $\sim(2 \mathrm{Mpc})^{3}$ part of the model cluster, the shock waves and perturbations in the ICM, caused by subcluster mergers, mix the enriched regions on shorter timescales (several hundred Myr), depending on the mass ratios and the merger scenario.

\subsubsection{Evolution of the mean metallicities}

In order to give a quantitative estimate of the enrichment, we present the evolution of the mean metallicities of our model clusters during the time interval $z=1$ to $z=0$ in Fig. 10 . The quantities given are the mean metallicities extracted from the X-ray weighted metal maps, weighted with the X-ray surface brightness maps. In Fig. 10 a region of $(2.5 \mathrm{Mpc})^{2}$ was taken into account, which corresponds to a typical galaxy cluster size. Whereas model cluster A and C show approximately a constant slope over the simulation time, model cluster B reveals a different behaviour. At $10 \mathrm{Gyr}$ an enhancement is visible because of a merger event, which increases the X-ray weighted 


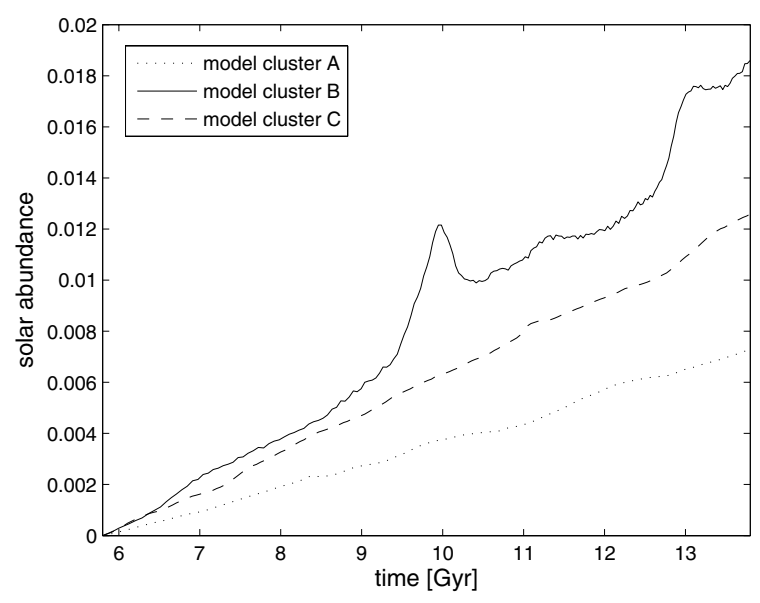

Fig. 10. Evolution of the X-ray weighted metallicity for all model clusters. The metal maps of the $(2.5 \mathrm{Mpc})^{2}$ region are weighted by X-ray surface brightness maps in the same region.

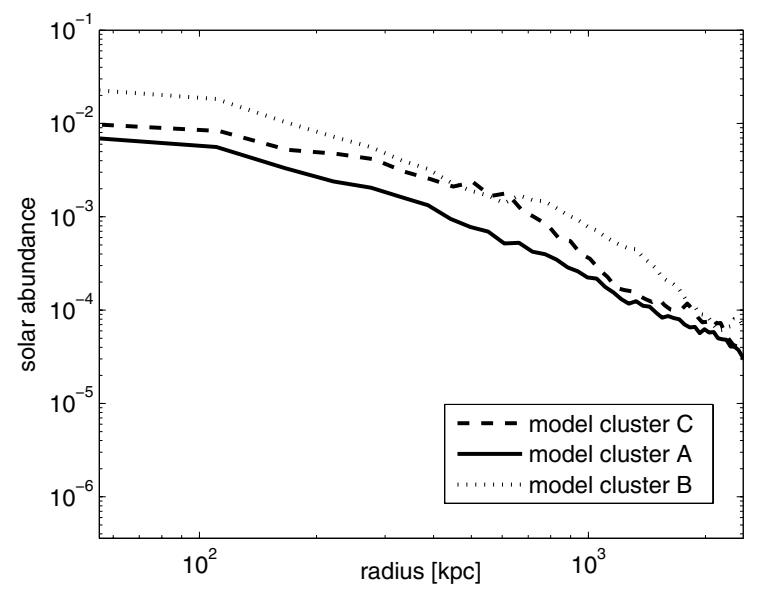

Fig. 11. Metallicity profiles of the three model clusters at $z=0$.

metallicities due to a temporary increase in density in the merger region. In the case of model cluster $\mathrm{B}$, the first maximum in the mean velocity (see Fig. 7) corresponds to the enhancement of the metallicity enrichment of model cluster B in Fig 10, which is related to a cluster merging event.

In model cluster B the increase in the number of galaxies in the simulation volume with $(5 \mathrm{Mpc})^{3}$ is stronger than in the model clusters $\mathrm{A}$ and $\mathrm{C}$. Therefore the increase in the enrichment rate can be explained as increase of number of galaxies with galactic winds in the simulation volume.

In Fig. 11 the metallicity profiles of the three model clusters at $z=0$ are shown. The centre of the $2 \mathrm{D}$ binning coincides in all cases with the maximum in density of the baryonic matter.

The central metallicity in model cluster B increases by an order of magnitude within $\sim 4 \mathrm{Gyr}$, ending at $z=0$. Metal profiles are easier to obtain from observations than 2D metal maps, but they have one major disadvantage: they do not show the inhomogeneities in the metal distribution of a galaxy cluster. The comparison of the metal profile of model cluster $B$ at the end of the simulation with the corresponding artificial X-ray weighted metal map in Fig. 13 shows this issue clearly. Due to the averaging of metals in rings centred around the emission centre of

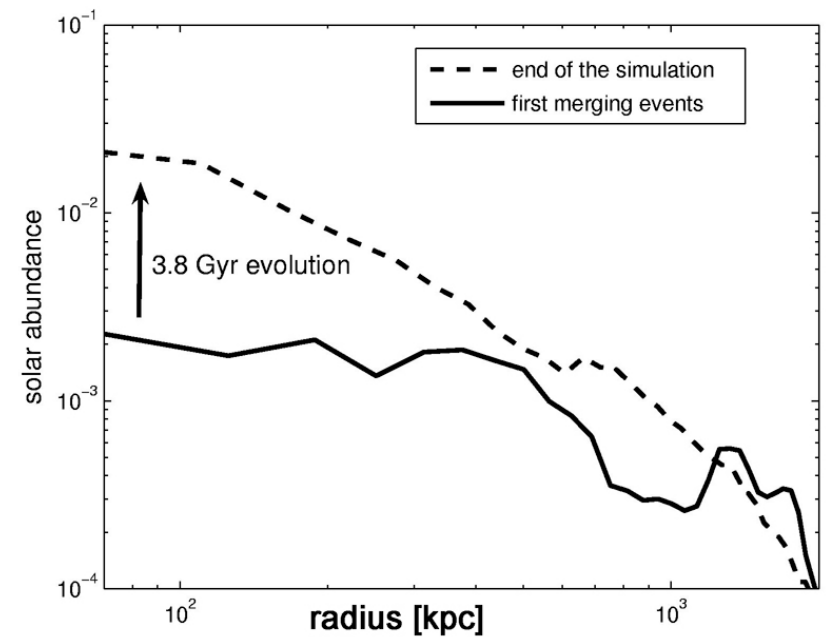

Fig. 12. Metallicity profile for model cluster B at the first merging events and at the end of the simulation.
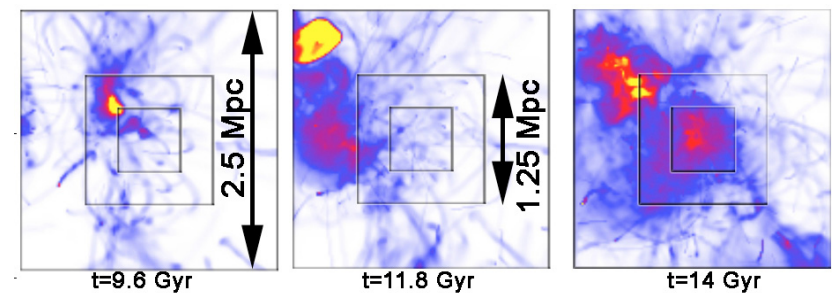

Fig. 13. X-ray weighted metal maps for model cluster B at different times. The movement of the metals with respect to the X-ray surface brightness centre (fixed at the image centre) due to several cluster mergers can easily be seen.

the ICM, the real maximum in the 2D metal maps is flattened out.

\subsection{Revealing the dynamical state through $X$-ray maps}

Galaxy clusters are the largest gravitationally bound systems in the universe and, as they are formed from huge amounts of DM, galaxies and ICM, it takes cosmological timescales to establish dynamical equilibrium. Galaxy clusters can be observed in many wavelengths to study the interaction of the different components. One outstanding challenge is the comparison of high resolution X-ray metal maps with galaxy-density maps. The combination of both observations will lead to a deeper insight into the interaction processes in galaxy clusters. In this section we want to present the capabilities of these combined observations to address questions about the dynamical state of a galaxy cluster.

If metals originate from galaxies, they are expelled by processes like ram-pressure stripping, galactic winds or direct kinetic redistribution of material by galaxy-galaxy interaction. Therefore they are connected to the trajectories of the galaxies. The relative velocity of the galaxies with respect to the ICM then leads to metal tracks, which can survive for several Gyr in the quieter outer regions of a galaxy cluster. In Fig. 14 X-ray weighted metal maps for model cluster B are 

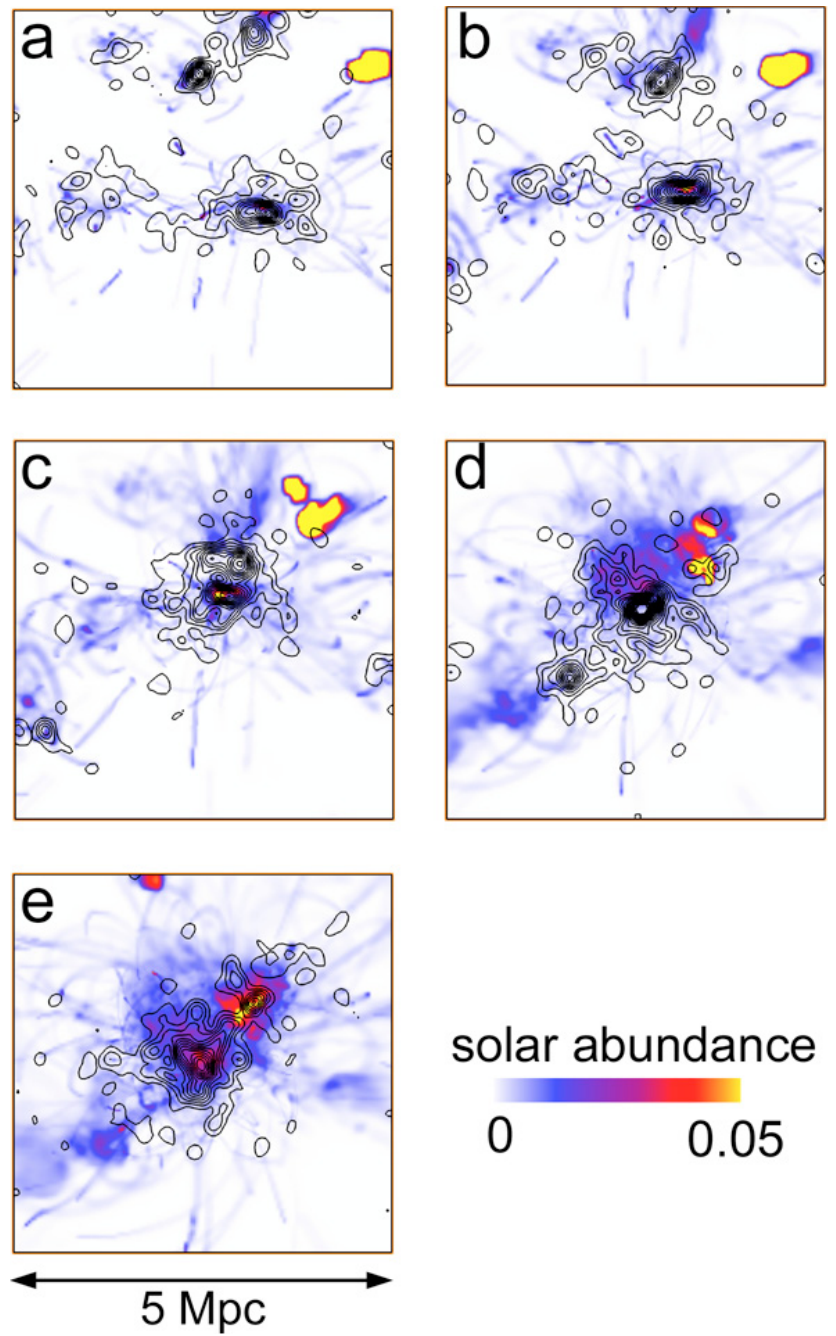

solar abundance

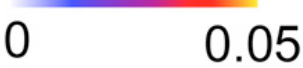

Fig. 14. X-ray weighted metallicity maps of model cluster B with galaxy-density isolines. Images a) to e) show the model cluster at different times, chosen in such a way that pre- and in situ merger evolutionary states are presented (image a to e shows about $2.5 \mathrm{Gyr}$ of evolution). The metallicity-gaps in front (relative to the direction of motion) of the substructures are clearly visible.

shown, with isolines representing the galaxy number density. Images (a) to (e) show different evolutionary states of model cluster B, the model cluster with many merger events. The images (a), (b) and (c) show the infall of a major subcluster from the top. Image (c) shows the cluster in the pre-merger phase. Along their way to the main cluster the galaxies lose metals and therefore there is a higher metallicity behind the subcluster than in front of them. In image (d) another subcluster falls into the main cluster (from the lower left). Again there is a higher metallicity behind the subcluster than in front of it. This results in a gap in the metal map, between the subcluster and the main cluster, just before the two structures merge.

In Fig. 15 two timesteps of the merging model cluster B are presented. The upper images show the cluster shortly before $(\sim 80 \mathrm{Myr})$ and shortly after $(\sim 60 \mathrm{Myr})$ the first passage of the subcluster through the main-cluster. In the lower panels (image (c) and (d)) magnifications of the central $(2.5 \mathrm{Mpc})^{2}$ cluster region are shown. Before the first encounter (image (c))

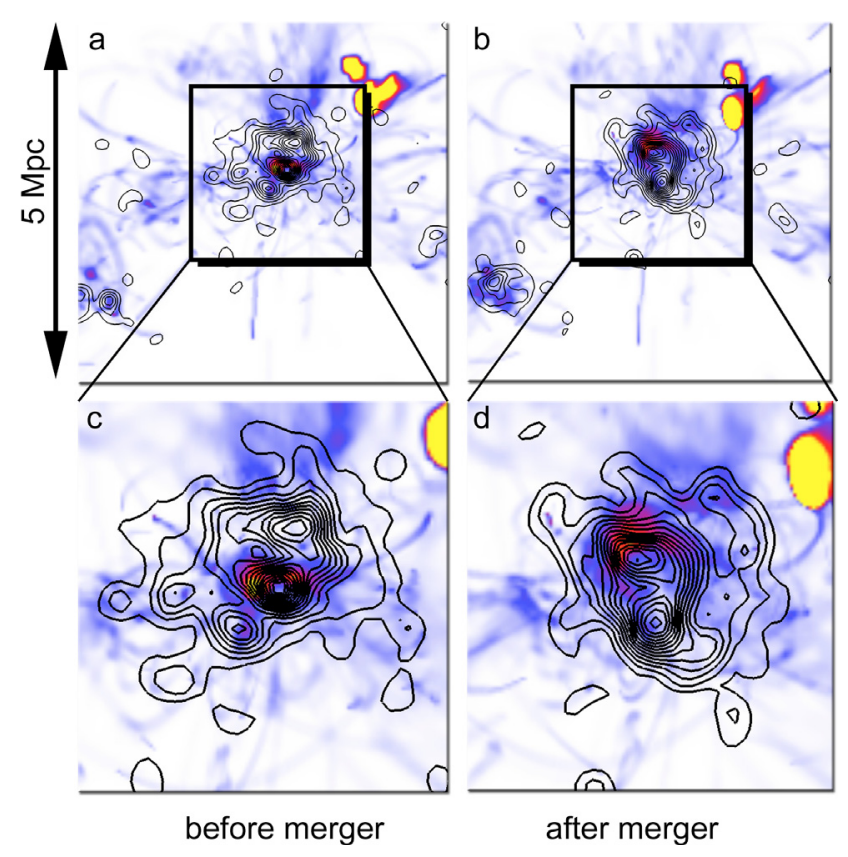

Fig. 15. Artificial X-ray weighted metallicity maps of model cluster B with galaxy-density isolines drawn in (the color bar is the same as in Fig. 14). The upper images show again the $(5 \mathrm{Mpc})^{2}$ region, whereas the lower images are the magnifications of the innermost grid $(2.5 \mathrm{Mpc})^{2}$. The metallicity gap before the first encounter of the subcluster with the main cluster is clearly visible. The main cluster is located in the centre of the image $\mathbf{c}$ ) and the gap lies above.

the absence of metals between the main- and the subcluster is clearly visible. In image (d) the subcluster with the corresponding galaxies went through the main cluster and therefore there is no metallicity gap between them anymore.

The combination of both observations (optical imaging and $\mathrm{X}$-ray) can reveal the dynamical state of a galaxy cluster very accurately, without any time consuming optical spectroscopic investigations. The fact that there is a strong gradient in the metallicity distribution in front of and behind the infalling subcluster, indicates the direction of motion of the galaxies and the pre- or post merger state of a galaxy cluster.

Of course, our simulated metallicity maps have resolutions not obtainable with present X-ray observations. Therefore Fig. 16 shows more realistic metal maps, including galaxy densities isolines. The right images give the same quantities as the left ones, but with a resolution of $9 \times 9$ pixels. The metallicity gap between the main- and subcluster in the upper images is present, in the high as well as in the low resolution image. In the lower images another subcluster falls into the main cluster from the lower left corner. Again the higher metallicity behind the subcluster, which moves towards the main cluster, is visible in both the low and high resolution metal maps.

\subsection{Comparison with observations: the bimodal clusters Abell 3921 and Abell 3528}

In order to test whether the metallicity gap between premerging structures in galaxy clusters are observable or not, we compared the three model clusters with observed galaxy 


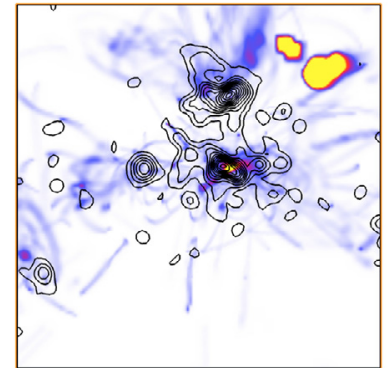

$5 \mathrm{Mpc}$
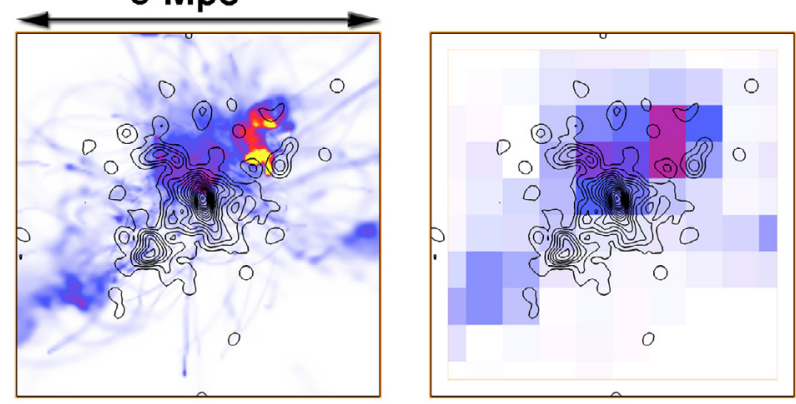

Fig. 16. X-ray weighted metallicity maps of model cluster B with galaxy-density isolines. The color bar is the same as in Fig. 14. The left images show the simulation resolution, the right images present the same metal maps with a resolution of $9 \times 9$ pixel.

clusters. Two systems in which metal gaps have been observed, and whose dynamical state has been studied in detail through a multi-wavelength analysis, are presented in the following: Abell 3921 and Abell 3528.

\subsubsection{Abell 3921}

Abell 3921 is a $R=2$, BM II cluster at $z=0.094$ (Katgert et al. 1998). Recent optical and X-ray analysis (Ferrari et al. 2005; Belsole et al. 2005) showed that its perturbed morphology, firstly revealed by ROSAT and Ginga observations (Arnaud et al. 1996), is due to an on-going merger of two main sub-structures: a main cluster centred on the Brightest Cluster Galaxy (BCG) (A3921-A), and a NW subcluster (A3921-B) hosting the second brightest cluster member. A3921-A is $\sim 5$ times more massive than A3921-B. The comparison of the optical and X-ray properties of A3921 (i.e. dynamical and kinematic properties of the cluster, optical and X-ray morphology, features in the ICM density and temperature maps) suggests that A3921-B is tangentially traversing the main cluster in the S-SE North direction. The two sub-clusters show a collision axis nearly perpendicular to the line of sight (Ferrari et al. 2005; Belsole et al. 2005).

Belsole et al. (2005) extracted the ICM metallicity in three different regions of A3921: the Eastern part of the main cluster (in which the ICM is less perturbed by the merging event), the hot bar detected on the temperature map, and the western region (see Fig. 5 of Belsole et al. (2005) and Fig. 17). Taking their result as best reference, the metallicity of the western region is significantly higher ( $\sim 90 \%$ significance level) than in the main region and in the hot bar of A3921.

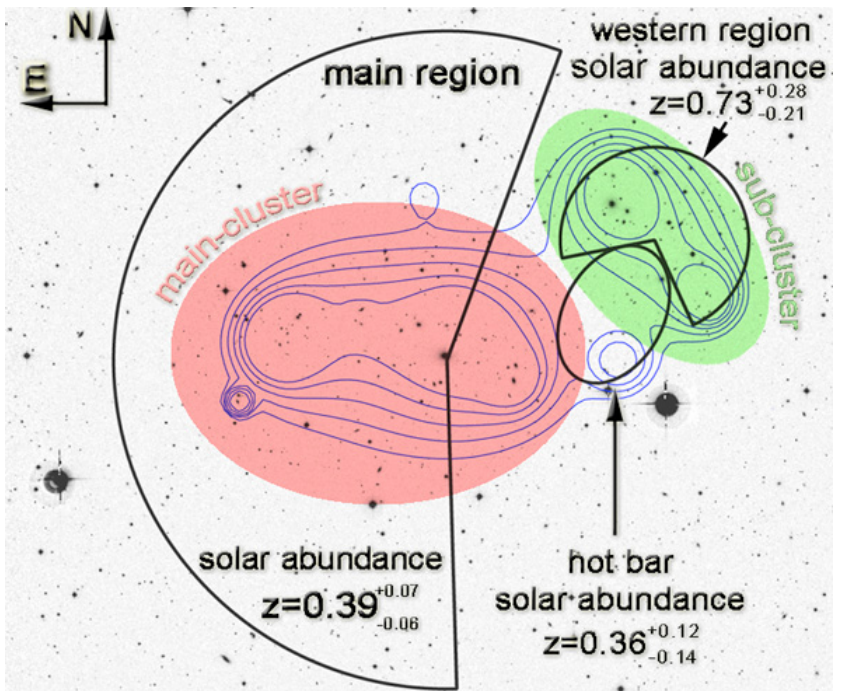

Fig. 17. Iso-density contours (blue) of the projected distribution of the red sequence galaxies (with $R_{\mathrm{AB}} \leq 19$ ) superimposed on the central $30 \times 22 \mathrm{arcmin}^{2}$ of the $R$-band image of A3921. Metallicity of the ICM (solar abundance) was determined from XMM data by Belsole et al. (2005) in three regions outlined by the black lines.

In Fig. 18 the metallicity distribution of model cluster B is shown. The insert gives in addition one ICM iso-density surface, which shows the positions of the main- and the subcluster. As mentioned above, metals, ejected by galactic winds and merger-driven starbursts, are located behind the subcluster. As galaxies in subclusters interact with lower relative velocities (several $100 \mathrm{~km} \mathrm{~s}^{-1}$ ), they show stronger enhancements in their SFRs than in the cluster central regions, where the relative velocities are of the order of $1000 \mathrm{~km} \mathrm{~s}^{-1}$. In addition the gas content of cluster galaxies, especially in the central regions, are lower due to ram-pressure stripping. In Abell 3921 the western region shows the highest metallicity, nearly twice of that of the main region. If our picture holds, the subcluster seems therefore to be in the pre-merger state.

This confirms that the previous hypothesis that A3921 is in the phases of merging (Ferrari et al. 2005, $t_{0} \sim \pm 0.3 \mathrm{Gyr}$ ) is correct, but we can now even refine the merging scenario by affirming that the closest cores encounter has not yet happened (in partial disagreement with the conclusions of Belsole et al. 2005). If a high resolution metal map were available, we would probably also be able to reconstruct the 3D-path of the subcluster A3921-B.

\subsubsection{Abell 3528}

Abell 3528 is a $R=1$, BM II Abell cluster at $z=0.053$ located in the core of the Shapley Concentration. X-ray observations (Raychaudhury et al. 1991; Schindler 1996) revealed a bimodal morphology, with two main clumps (A3528-N and A3528-S) aligned in the North/South direction and separated by $\sim 14 \operatorname{arcmin}\left(0.9 h_{70}^{-1} \mathrm{Mpc}\right)$. Further optical, X-ray and radio analysis (Bardelli et al. 2001; Baldi et al. 2001; Donnelly et al. 2001; Venturi et al. 2001) indicated that A3528 is a premerging system, since: a) the galaxies in the two clumps are 


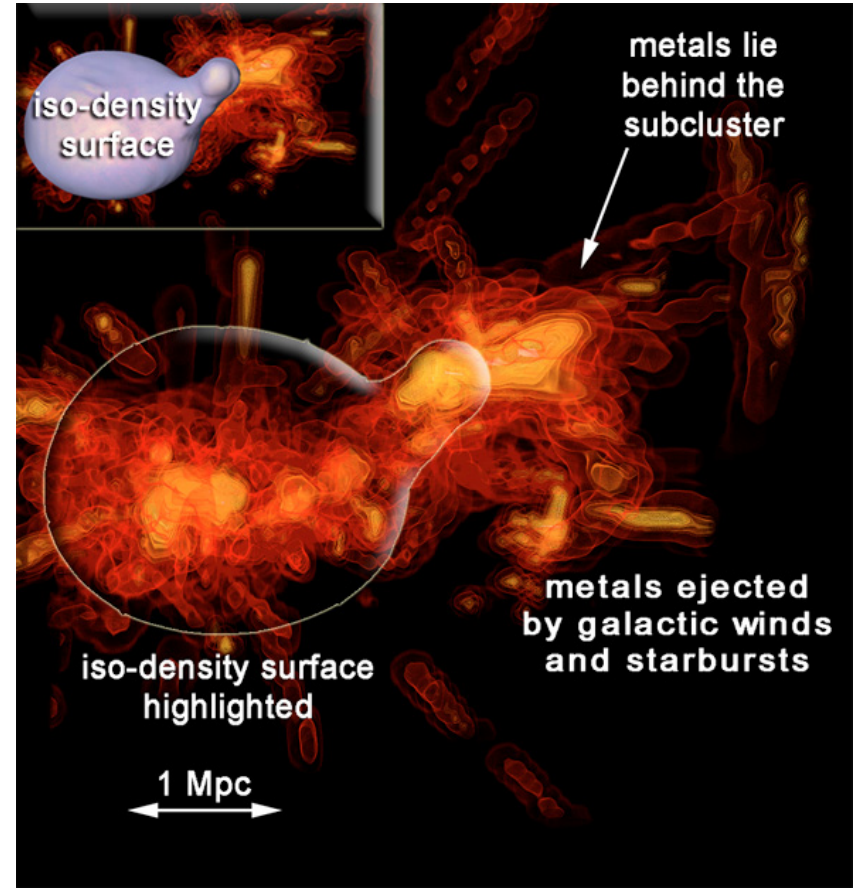

Fig. 18. The 3D metal distribution of model cluster B and the edge of the ICM density isosurface (highlighted by the insert) are shown. The subcluster has higher metallicities, due to a merger-driven starburst, and the offset of the metals with respect to the subcluster is visible. In addition the gap in metallicity, indicating the pre-merger state, can be seen clearly.

not far from the virial equilibrium each; b) the merging effects on the galaxy population are not yet evident, neither at optical nor at radio wavelengths; c) no diffuse extended radio emission has been detected; and d) the ICM surface brightness distribution of the two clumps is still azimuthally symmetric, with evidences for cool cores, and the gas located between the two clumps is only marginally hotter $(\sim 15 \%)$.

Through XMM observations of A3528, Gastaldello et al. (2003) reached different conclusions on the dynamical state of this cluster, assessing that in fact A3528 is an off-axis postmerging system. They conclude that the absence of any evidence of shock heated gas both in their temperature and in their density map is due to a wrong initial hypothesis, i.e. the two clumps cannot be in a pre-merger phase. By combining this result with: a) the absence of any strong merging signatures in the X-ray data, despite the relatively small separation of the two clusters, and b) the optical light distribution mostly concentrated in the northern clump, Gastaldello et al. (2003) suggest that the closest core encounter between A3528-N and A3528-S happened about 1-2 Gyr ago, with a high impact parameter. In agreement with the previous X-ray studies (ROSAT and ASCA data, Donnelly et al. 2001), their analysis confirms that the two main clumps in X-ray have a relaxed structure, a centrally peaked surface brightness, and cooler cores.

The comparison of the metallicity map obtained from XMM data (Gastaldello et al. 2003) with the results of our numerical simulations can help to shed light on the dynamical state of A3528. In Fig. 19 the isodensity contours of the galaxy number and the metallicity of the ICM in several regions are

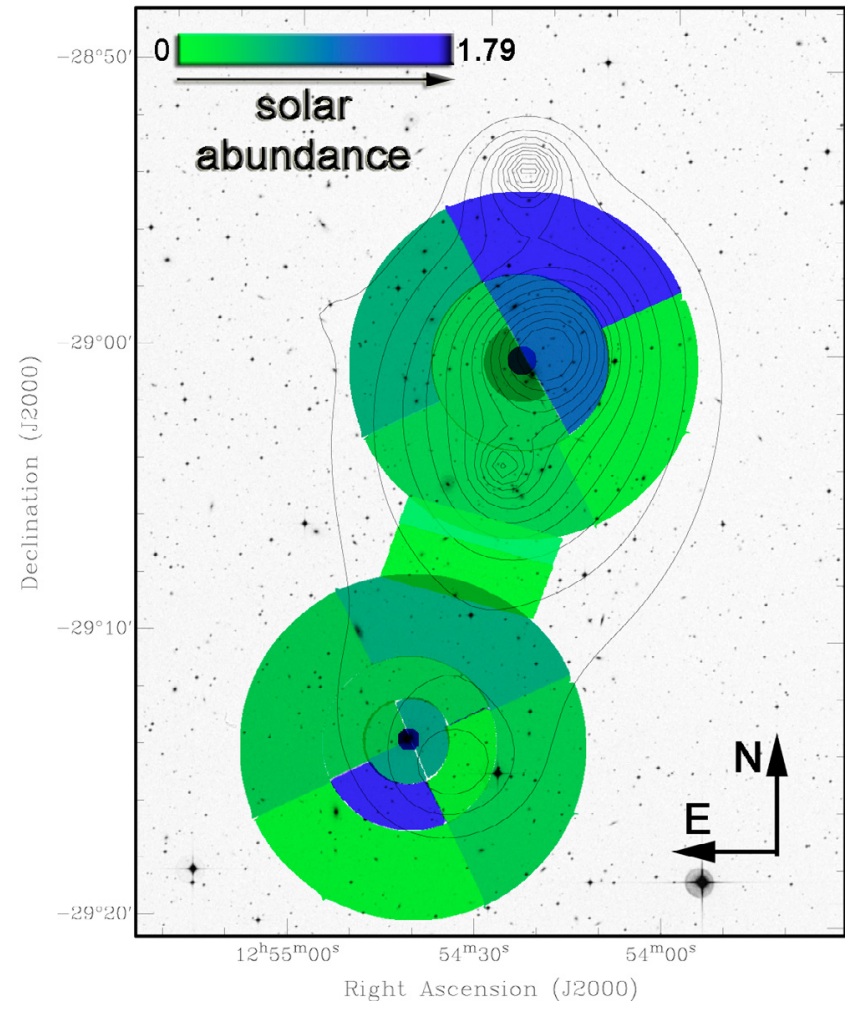

Fig. 19. Iso-density contours (black) of the projected distributions of galaxies $(13 \leq B \leq 19.5)$ in the A3528 central field. The abundances in the annuli surrounding the two main sub-clusters (A3528-N and A3528-S) and in the region between them are shown in color (Gastaldello et al. 2003).

shown. The isodensity map has been built from the APM catalogue (13 $\leq B \leq 19.5)$ on the basis of a multi-scale approach (see Ferrari et al. 2005, for more details). The highest abundances are observed in the SE of the core of A3528-S and in the NW region of A3528-N, while a lower metallicity characterises the region between the two clumps. Following our simulations, these results would suggest that A3528-N and A3528-S are in reality in a pre-merger phase, with a collision axis along the $\mathrm{SE} / \mathrm{NW}$ direction, in agreement with the first hypothesis about the dynamical state of A3528 (Bardelli et al. 2001; Baldi et al. 2001; Donnelly et al. 2001; Venturi et al. 2001). It is however clear that, due to the large errors in the abundance determination (Gastaldello et al. 2003), a higher statistics in the iron line would be useful to confirm the pre-merger scenario.

\subsection{Metal enrichment before $z=1$}

As we start simulating the ICM of our model clusters at $z=1$, the question arises what the fractions of metals ejected by galactic winds and starbursts are in earlier epochs. We adress this question by applying our wind and starburst routines to the semi-numerical galaxies in the redshift range of $z=4$ to $z=0$. As we do not have the knowledge of the ICM properties before $z=1$, we do not suppress quiet galactic winds by pressure of the ICM on galaxies in the central part of the galaxy cluster. In addition we sample the ejected metals along the trajectories of the galaxies on a $(20 \mathrm{Mpc})^{3}$ co-moving grid to get an idea 


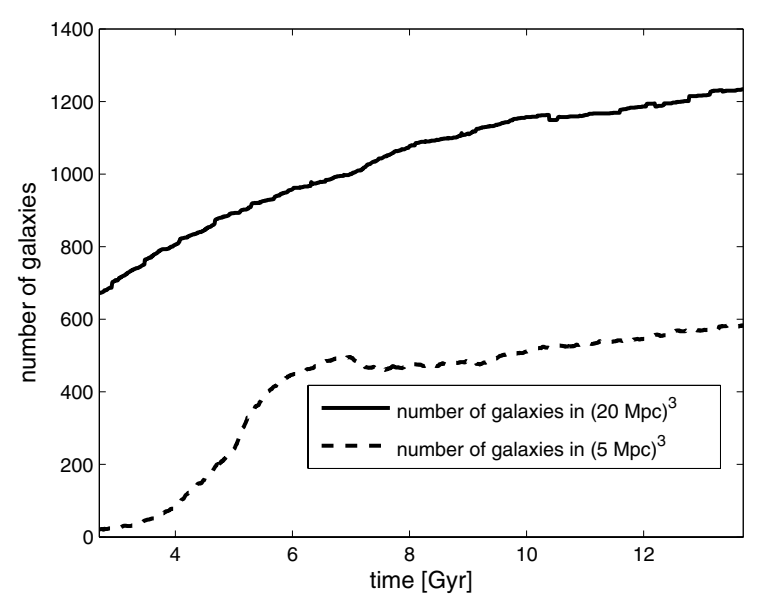

Fig. 20. Evolution of the number of galaxies in the $(20 \mathrm{Mpc})^{3}$ and $(5 \mathrm{Mpc})^{3}$ simulation volume starting from $z=4(\sim 1.5 \mathrm{Gyr})$ and ending at $z=0(\sim 13.8 \mathrm{Gyr})$ for model cluster $\mathrm{C}$ as an example is shown.

of the metal-distribution from the early epochs. The metals are only put into cells at the positions of the galaxies without assigning any velocities. In this very simple approach we sample all the metals on a $128^{3}$ grid. This procedure helps us to see where galaxies lose their metals, in the centre or in the outskirts. In Fig. 20 the evolution of the number of galaxies in the $(20 \mathrm{Mpc})^{3}$ and $(5 \mathrm{Mpc})^{3}$ co-moving simulation volume starting from $z=4$ for model cluster $\mathrm{C}$ is shown. As the cluster forms around $z=1(\sim 5.8 \mathrm{Gyr})$, the increase of galaxies in the central $(5 \mathrm{Mpc})^{3}$ region of the cluster is clearly visible.

The amount of metals ejected by all galaxies having a quiet galactic wind or a starburst as a function of time is shown in Fig. 21. The black lines give the amount of metals in the $(20 \mathrm{Mpc})^{3}$ simulation volume, whereas the red lines give the same quantity in the $(5 \mathrm{Mpc})^{3}$ volume. The different processes (galactic winds and starbursts) are plotted separately. Starbursts happen more often in the outskirts of the galaxy cluster, where the galaxies move in groups towards the cluster. Before $z=1$ merger-driven starbursts are the dominating enrichment process in comparison to galactic winds in model cluster C. In the smaller volume, representing the cluster, only two strong merger-driven starbursts happen since $z=4$. Therefore winds dominate as enrichment process in the $(5 \mathrm{Mpc})^{3}$ volume.

The amount of metals ejected by galactic winds and starbursts before and after $z=1$ is given Table 2 . The first trend is that starbursts occur more often in the outskirts of the cluster, but they do not play an important role for enriching the central $(5 \mathrm{Mpc})^{3} h^{-1}$ region. Note that in this approximation the ejected gas does not follow the potential (time dependent) as we do it in the hydrodynamical approach derived above. This is a good approximation as the infalling gas does not fall right to the dense centre, but it is stopped by the ICM at large radii. Therefore enriched outer regions (above 5-7 Mpc distance from the cluster centre) will not have entered the cluster centre yet. Most starbursts before $z=1$ happen in these outer regions and therefore they contribute only little to the overall metal abundance in the central cluster region $(r<3 \mathrm{Mpc})$. As the number of galaxies with mass losses by galactic winds increases with a higher rate in the $(5 \mathrm{Mpc})^{3} h^{-1}$ volume than in the $(20 \mathrm{Mpc})^{3} h^{-1}$, see e.g.

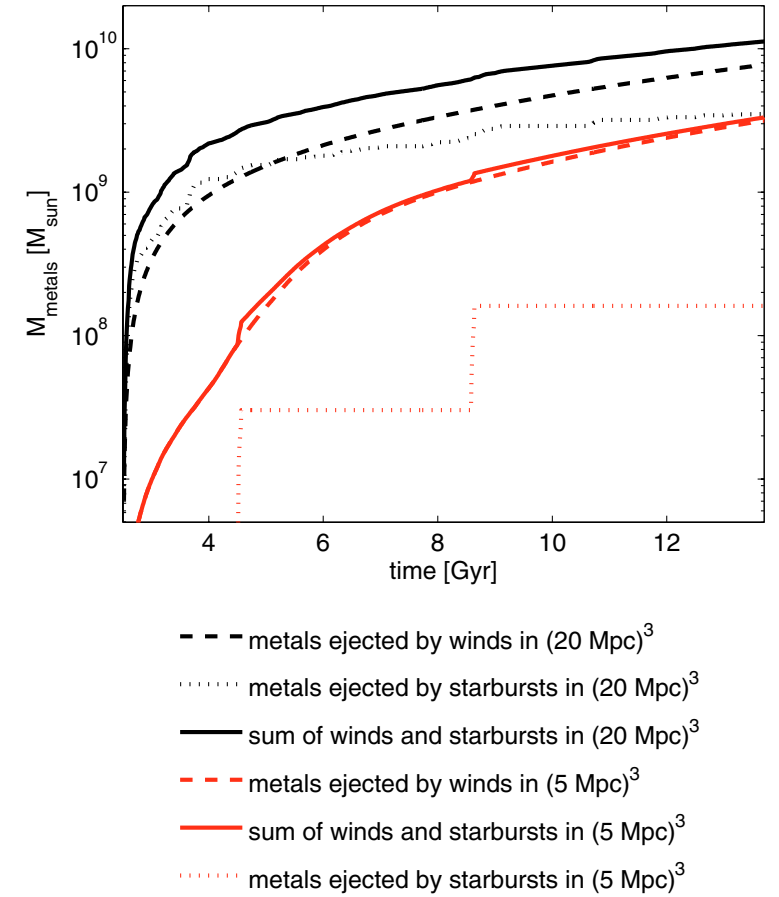

Fig. 21. Amount of metals ejected into the ICM by galactic winds and starbursts starting at $z=4$ for model cluster $\mathrm{C}$. The black lines show the amount in the $(20 \mathrm{Mpc})^{3} h^{-1}$ and the red lines in the $(5 \mathrm{Mpc})^{3} h^{-1}$ simulation co-moving volume.

Fig. 20, the amount of metals ejected by quiet galactic winds in the redshift interval $z=1$ to $z=0$ is higher in the $(5 \mathrm{Mpc})^{3} h^{-1}$ volume. This trend is even more pronounced in the merging cluster. Galactic winds are dominating the enrichment in comparison to starbursts. But this has an intrinsic uncertainty, i.e. the metallicity of the starburst ejecta, especially if the metallicity of the ejected gas in high redshift starbursts is higher than in the semi-numerical model.

In Fig. 22 the distribution of metals in the $(20 \mathrm{Mpc})^{3}$ simulation volume at $z=1$ and and at $z=0$ is shown. The amount of metals is calculated as described before, without the hydrodynamic calculation of the ICM. The distribution of starbursts in the outskirts of the cluster is clearly visible, whereas the galactic winds are the dominant enrichment process in the central regions. At $z=1$ the model cluster is starting to form. Therefore not many galaxies, with a galactic wind, are in the cluster region. At the end of the simulation at $z=0$ the picture changes: winds have enriched the central region. In Fig. 23 we compare the metal distribution calculated with the simple approach without ICM of this section and with our combined $N$-body/hydrodynmic approach. The distribution is given at $z=0$ for model cluster $\mathrm{A}$, the most massive one. In the simple approach the metals are peaked around the centre and not as widely distributed as in the $N$-body/hydrodynmic approach (see images (a) and (b) in Fig. 23). The pressure of the ICM on the galaxies in the very central region $(\sim 1 \mathrm{Mpc}$ around the cluster centre) does suppress galactic winds (image b). This is not the case in the simple approach (image a), where the metals from galactic winds are peaked around the centre. Although the approach in this section is very simple, the elongated metal 
Table 2. Amount and fractions of metals ejected in the redshift intervals $z \in[4,1]$ and $z \in[1,0]$.

\begin{tabular}{ccccccc}
\hline \hline $\begin{array}{c}\text { Co-moving } \\
\text { volume }\end{array}$ & $\begin{array}{c}\text { Model } \\
\text { cluster }\end{array}$ & $\begin{array}{c}\text { All metals } \\
\text { ejected } z \in[4,0] \\
{\left[10^{9} M_{\odot}\right]}\end{array}$ & $\begin{array}{c}\text { Ejected by } \\
\text { galactic winds } \\
z \in[4,1]\end{array}$ & $\begin{array}{c}\text { Ejected by } \\
\text { galactic winds } \\
z \in[1,0]\end{array}$ & $\begin{array}{c}\text { Ejected by } \\
\text { starbursts } \\
z \in[4,1]\end{array}$ & $\begin{array}{c}\text { Ejected by } \\
\text { starbursts } \\
z \in[1,0]\end{array}$ \\
\hline$(20 \mathrm{Mpc})^{3} h^{-1}$ & $\mathrm{~A}$ & 14.2 & $29 \%$ & $48 \%$ & $14 \%$ & $9 \%$ \\
$(20 \mathrm{Mpc})^{3} h^{-1}$ & $\mathrm{~B}$ & 14.7 & $20 \%$ & $42 \%$ & $17 \%$ & $21 \%$ \\
$(20 \mathrm{Mpc})^{3} h^{-1}$ & $\mathrm{C}$ & 11.3 & $18 \%$ & $51 \%$ & $16 \%$ & $15 \%$ \\
\hline$(5 \mathrm{Mpc})^{3} h^{-1}$ & $\mathrm{~A}$ & 5.2 & $24 \%$ & $75 \%$ & $0 \%$ & $1 \%$ \\
$(5 \mathrm{Mpc})^{3} h^{-1}$ & $\mathrm{~B}$ & 2.7 & $13 \%$ & $87 \%$ & $0 \%$ & $0 \%$ \\
$(5 \mathrm{Mpc})^{3} h^{-1}$ & $\mathrm{C}$ & 3.3 & $11 \%$ & $84 \%$ & $1 \%$ & $4 \%$ \\
\hline
\end{tabular}
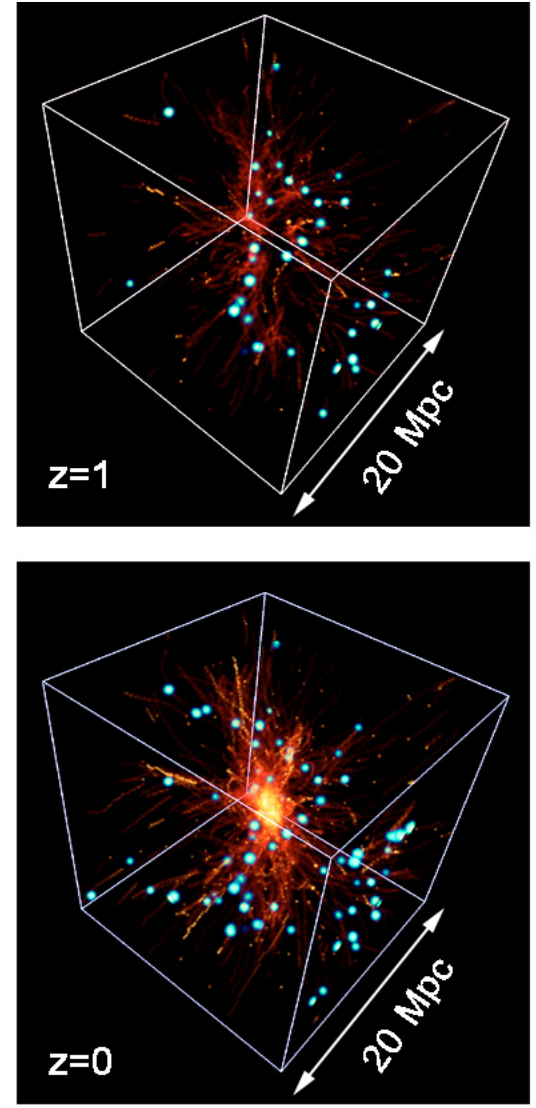

starbursts - green galactic winds - red

Fig. 22. The distribution of metals in the $(20 \mathrm{Mpc})^{3}$ simulation volume at $z=1$ and and at $z=0$. The amount of metals is calculated as described in the text, without the hydrodynamic calculation of the ICM. The green colour shows the metals ejected by starbursts, whereas the red colour indicates metals ejected by galactic winds.

stripes can be found in both approaches. Note that the mass resolution of the $N$-body simulation has a limit of $1 \times 10^{11} M_{\odot}$ (baryonic and non-baryonic). Galactic winds of spiral galaxies seem not to be responsible for all of the observed metallicities in the ICM. In the case of starbursts the mean uncertainty in our model is the metallicity of the ejected gas, especially at high redshifts. Although we do not resolve dwarf spiral galaxies, which will contribute to the overall enrichment of the ICM, the uncertainty in the metallicities in the outflows of high

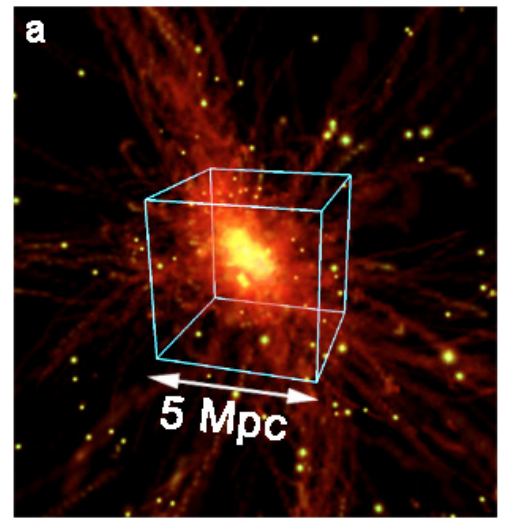

b

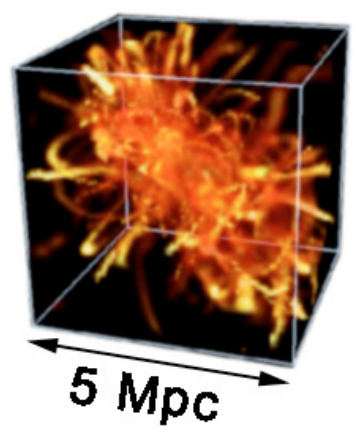

Fig. 23. Distribution of metals for model cluster $\mathrm{A}$ at $z=0$. Image a shows the result of metal ejection by galactic winds and starbursts with the simple approach used in this section. Image b) gives the same quantity from the combined $N$-body/hydrodynamic simulation.

redshift starbursts can alter the results most. Therefore the estimate given here represents a lower limit.

There are some differences of our approach compared to other work in this field (e.g. de Lucia et al. 2004; Aguirre et al. 2005; Springel \& Hernquist 2003; Tornatore et al. 2004). In de Lucia et al. (2004) ( $N$-body simulations with a semianalytical galaxy evolution model) there are three transport processes modelled: the ejection, retention and wind model. Whereas the wind model enriches directly the ICM, the retention model enriches the halo of the galaxy (cooling is included) and the ejection model enriches the ICM with metals, which are then reincorporated into the host halo in a given time interval. Their result is that the ejection model contributes the highest fraction of metals to the ICM at present epochs. The observed 
metallicities in the ICM (taken from X-ray observations) are compared with the sum of metals in the halos of galaxies and the ICM in their simulation. Our wind model takes into account only gas which is not gravitationally bound to the galaxy furthermore and is therefore heated by the ICM to temperatures, in which X-rays are emitted in an energy range accessible to current X-ray telescopes. Although supernovae driven outflow enrich the hot gas in the halo of a galaxy, only a minor fraction of enriched gas will be ejected into the ICM, if the SFRs are low (about $1 M_{\odot} / \mathrm{yr}$ ). The major fraction will not reach the escape velocity, will cool radiatively cool and become then a part of the cold disk gas again.

In Aguirre et al. (2005) combined $N$-body/SPH simulations were done to confront cosmological simulations with observations of intergalactic metals. Their applied wind model and cooling description is not able to reproduce the observed amount of metals in the intergalactic medium (IGM). The metallicity map given in Springel \& Herquist (2003) does not distinguish between hot IGM gas and gas in the halo of the galaxies. Tornatore et al. (2005), combined $N$-body/SPH simulations on galaxy cluster scales and give profiles of the Fe abundance in simulated galaxy clusters. Although the profiles do not match the spatial distribution of metals observed in galaxy clusters, the overall amount of metals seems to be reproduced. Unfortunately X-ray emission weighted metal maps are not given to see, if the metals would be observable in X-ray observations.

A major difference in our model compared to de Lucia et al. (2004), Springel \& Hernquist (2003), Aguirre (2005) and Tornatore et al. (2004) is the calculation of X-ray emission weighted metal maps. As we are interested in a direct comparison with X-ray observations we treat the ICM hydrodynamically (including radiative cooling (Sutherland \& Dopita 1993)) and extract artificial X-ray maps from our simulations to compare them directly with real X-ray observations of galaxy clusters. This allows us to conclude that galactic winds and starbursts are in general not sufficient to enrich the ICM of galaxy clusters up to about 0.3 solar abundance in metallicity, which is observed in galaxy clusters in the X-ray regime. Additional processes like ram-pressure stripping (see Domainko et al. 2005), stripping of hot halo gas or jets of AGN are not taken into account in this work. Their contribution is addressed in Domainko et al. (2005) and subsequent papers.

\subsection{X-ray weighted metal maps of our model clusters as observed with Chandra, XMM-Newton and XEUS}

As mentioned above, observations are always limited by the number of X-ray photons. The X-ray weighted metal maps obtained from our simulations have resolutions which cannot be reached by state of the art observations with instruments like Chandra or XMM-Newton. In Fig. 24 four X-ray weighted metal maps of model cluster B at $z=0$ are shown. Each map has a different resolution, reflecting the sensitivity of different X-ray instruments. The last map has the resolution of the simulation. Assuming that a cluster resides at a given distance
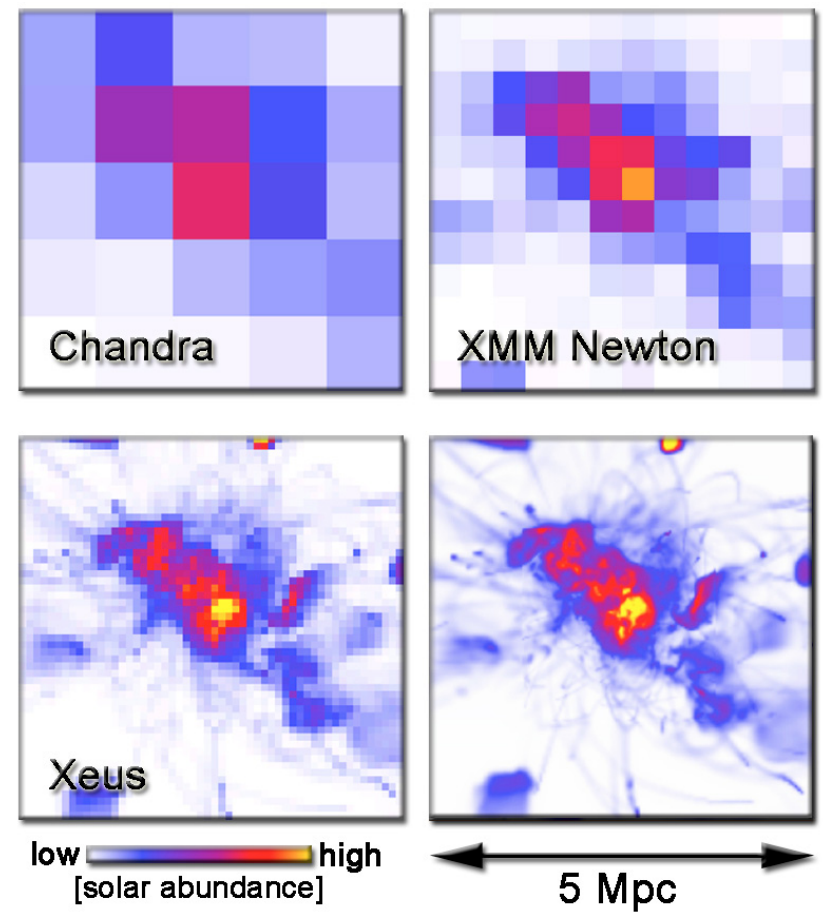

Fig. 24. X-ray weighted metal maps as they would be observed with Chandra, XMM-Newton and the next generation X-ray telescope XEUS for a given exposure time. Lower right image: the real metal map. Different sensitivity of the different missions yield different resolutions of the metal map.

which enables us to extract a $5 \times 5$ pixel $X$-ray weighted metal map with the X-ray telescope Chandra, XMM-Newton would be able to obtain a $12 \times 12$ pixel metal map within the same exposure time. This is due to the fact that XMM-Newton has a six times higher sensitivity than Chandra. XEUS is planned to be a follow-up mission of XMM-Newton, which will have about a sensitivity 20 times higher than XMM-Newton. For diffuse low surface brightness objects, like galaxy clusters, the effective area is a major issue, therefore for a reasonable observation time, Chandra maps will have lower resolution than XMM-Newton. With XEUS we will be able to resolve for the first time the metal distribution in the ICM on the scales of single galaxies in nearby clusters.

\subsection{On the survival timescales of inhomogeneities in the metallicity distribution}

As shown in the X-ray weighted metal maps, metallicity inhomogeneities can survive over long periods. The timescales depend strongly on the environment of the enriched regions in the ICM. If a highly enriched region is situated in the central part of a merging cluster, the metals will be spread quickly over huge volumes, due to the internal kinematics of the ICM. Perturbations propagating from the central regions into the outer parts blur enriched regions on shorter timescales, than in the outskirts (the low dense environment of a galaxy cluster). To give an upper limit of the survival timescale of these inhomogeneities, we investigated a stripe of enriched ICM, caused by a galaxy having a quiet galactic wind. In Fig. 25 two X-ray 


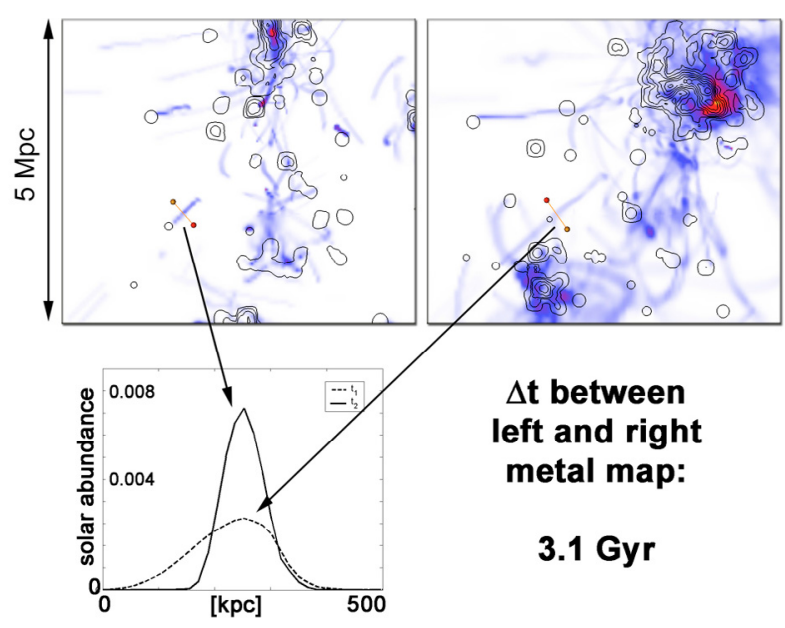

Fig. 25. Two metal maps of model cluster B for two different times, 3.1 Gyr of evolution between them. Along the red line the metal distribution is integrated and plotted.

weighted metal maps for model cluster $B$ are given, with a time difference of 3.1 Gyr. In the metal maps we measured a one dimensional metal distribution highlighted by two lines. Below the two metal maps the metallicity distribution as a function of space is shown for the two timesteps. As the enriched region diffuses, the maximum decreases by more than $50 \%$. If we integrate over the 2D distributions and compare them, the overall metallicity decreases in this region by a factor of 0.12 . The explanation for this decrease in the metallicity is the increase of density in the same region in the same time interval by a factor of 0.14 . The not previously enriched ICM coming from other mergers partly mixes with the enriched region.

Of course this effect depends strongly on the environment. In the innermost parts of a galaxy cluster metals are mixed with the ICM on shorter timescales, i.e. within several $100 \mathrm{Myr}$, whereas these inhomogeneities should be observable in the outer parts over several Gyr. In strongly merging galaxy clusters the timescales should be shorter than in relaxed massive clusters. This trend is noticeable in our simulations as well.

\section{Summary and conclusions}

We use combined $N$-body/hydrodynamic calculations with semi-numerical galaxy modelling to investigate galactic winds and starbursts as an enrichment process of the intra-cluster medium (ICM). The mass-loss rates by quiet galactic winds for spiral galaxies are calculated with a code developed by Breitschwerdt et al. (1991). The terminus mass loss rate in our simulations means galactic matter, which is not gravitationally bound to the originating galaxy anymore. As this enriched matter is then part of the ICM, we include it into our hydrodynamic ICM simulations. We then investigate the spatial distribution of the ejected matter and give X-ray surface brightness, X-ray emission weighted temperature and metal maps to study the dynamical state of the model galaxy clusters. We find that

- there is a difference between the strength of the enrichment by galactic winds and starbursts in merging and in non-merging model clusters. Merging systems show more galaxy-galaxy interactions, which cause more starbursts. On the other hand galactic winds can be suppressed by the pressure of the ICM onto the galaxies in relaxed clusters. As relaxed systems have more ICM to enrich, galactic winds are less effective. In our simulations we find that galactic winds and starbursts can contribute up to $5 \%$ (merging cluster) to the observed metallicities in galaxy clusters in the redshift interval from $z=1$ to $z=0$ and up to $15 \%$ in the redshift interval from $z=4$ to $z=1$.

- X-ray surface brightness, X-ray emission weighted temperature and metal maps can give a deeper insight into the dynamical state of a galaxy cluster. Temperature and metal maps are harder to obtain in observations (limited by the number of X-ray photons), but they yield a lot of information. We show that especially the metal maps can help to distinguish between the pre- or post merger state of a galaxy cluster. Pre-mergers have a metallicity gap between the subclusters, post-mergers have a high metallicity between subclusters. This holds also if the resolution is reduced to $9 \times 9$ pixels in our artificial X-ray weighted metallicity maps, which cover an area of $(5 \mathrm{Mpc})^{2}$.

- The inhomogeneities which are introduced by galactic winds and starbursts into the ICM can survive for a long time. In the outskirts of a galaxy cluster, where the variation in the density and the velocity field of the ICM is less than in the inner parts, we find that inhomogeneities can survive up to several Gyr. The timescales for the inhomogeneities are different for merging and non-merging galaxy clusters, because of mixing by shocks, introduced by major merger events (in the ICM).

- Metal enrichment of the ICM by galactic winds before $z=1$ is not as strong as after $z=1$ in the cluster centre $\left((5 \mathrm{Mpc})^{3} h^{-1}\right.$ volume $)$, whereas for merger-driven starbursts there is no noticeable difference. Before $z=1$ starbursts enrich the ICM on large scales at a comparable rate as galactic winds. As merger-driven starbursts happen more often in the outskirts of clusters, they enrich the outer (larger $r \sim 3 \mathrm{Mpc}$ ) regions of galaxy clusters. Galactic winds, on the other hand occur throughout the whole cluster, except for the densest innermost regions, where they might be suppressed by the high ICM pressure.

Note that the metal maps in this paper are directly comparable to observed metal maps, as we only take ejected matter into account for the enrichment processes, which would be observable in X-rays. The observed metallicities in galaxy clusters are one order of magnitude higher than in our simulations. We believe that galactic winds are not able to produce the total observed amount of metals in the ICM. Additional processes like ram-pressure stripping, hot halo gas stripping, jets of AGNs or intra-cluster supernovae obviously play an important role as well. A major uncertainty are the metallicities of the ejecta of high redshift starbursts, which will be investigated in an upcoming paper.

Acknowledgements. The authors are grateful to the anonymous referee for his/her criticism that helped to improve the paper. The authors acknowledge the Austrian Science Foundation (FWF) through grant number P15868, UniInfrastrukturprogramm 2004 the bm:bwk 
"Konsortium Hochleistungsrechnen", the bm:bwk Austrian Grid (Grid Computing) Initiative, the Austrian Council for Research and Technology Development and the support of the European Commission's Research Infrastructures activity of the Stucturing the European Research Area programme, contract number RII3-CT-2003506079 (HPC-Europa). In addition the authors acknowledge the DFG

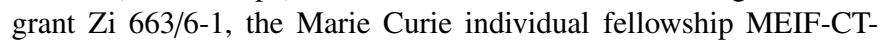
2003-900773 (CF) and the European Commission through grant number HPRI-CT-1999-00026 (the TRACS Program at EPCC). Edmund Bertschinger and Rien van de Weygaert are acknowledged for providing their constrained random field code and Joshua Barnes and Piet Hut for their tree code. The authors would like to thank Sabine Kreidl for many useful discussions.

\section{References}

Aguirre, A., Schaye, J., Hernquist, L., et al. 2005, ApJ, 620, L13

Arnaud, M., Rothenflug, R., Böhringer, H., Neumann, D., \& Yamashita, K. 1996, UV and X-ray Spectroscopy of Astrophysical and Laboratory Plasmas: Proc. of the Eleventh Colloq. on UV and X-ray, held on May 29-June 2, 1995, Nagoya, Japan (Universal Academy Press), Frontiers Science Series, 15, 163

Baldi, A., Bardelli, S., \& Zucca, E. 2001, 324, 509

Bardelli, S., Zucca, E., \& Baldi, A. 2001, MNRAS, 329, 387

Barnes, J., \& Hut, P. 1986, Nature, 324, 446

Belsole, E., Sauvageot, J.-L., Pratt, G. W., \& Bourdin, H. 2005, A\&A, 430,385

Breitschwerdt, D., McKenzie, J. F., \& Völk, H. J. 1991, A\&A, 245, 79

Colella, P., \& Woodward, P. R. 1984, J. Comp. Phys., 54, 174

De Young, D. S. 1978, ApJ, 223, 47

De Lucia, G., Kauffmann, G., \& White, S. D. M. 2004, MNRAS, 349, 1101

Domainko, W., Gitti, M., Schindler, S., \& Kapferer, W. 2004, A\&A, 425, L21

Domainko, W., Mair, M., Kapferer, et al. 2005, [arXiv: astro-ph/0507605]

Donnelly, R. H., Forman, W., Jones, C., et al. 2001, ApJ, 562, 254
Ferrari, C., Benoist, C., Maurogordato, S., Cappi, A., \& Slezak, E. 2005, A\&A, 430, 19

Fukazawa, Y., Kawano, N., \& Kawashima, K. 2004, ApJ, 606, L109

Furusho, T., Yamasaki, N. Y., \& Ohashi, T. 2003, ApJ, 596, 181

Gastaldello, F., Ettori, S., Molendi, S., et al. 2003, A\&A, 411, 21

Gnedin, N. Y. 1998, MNRAS, 249, 407

Gunn, J. E., \& Gott, J. R. III 1972, ApJ, 176, 1

Hayakawa, A., Furusho, T., Yamasaki, N. Y., Ishida, M., \& Ohashi, T. 2004, PASJ, 56, 743

Heckman, T. M., Lehnert, M. D., Strickland, D. K., \& Armus, L. 2000, APJS, 129, 493

Heckman, T. M. 2003, Rev. Mex. Astron. Astrofis., 17, 47

Kapferer, W., Knapp, A., Schindler, S., Kimeswenger, S., \& van Kampen, E. 2005, A\&A, 438, 87

Katgert, P., Mazure, A., den Hartog, R., et al. 1998, A\&AS, 129, 399

Martin, C. L. 1999, ApJ, 513, 156

Mo, H. J., Mao, S., \& White, S. D. M. 1998, MNRAS, 295, 319

Raychaudhury, S., Fabian, A. C., Edge, A. C., Jones, C., \& Forman, W. 1991, MNRAS, 248, 101

Ruffert, M. 1992, A\&A, 265, 82

Sanders, J. S., Fabian, A. C., Allen, S., \& Schmidt, R. W. 2004, MNRAS, 349, 952

Schindler, S. 1996, MNRAS, 280, 309

Schindler, S., et al. 2005, A\&A, 435, L25

Schmidt, R. W., Fabian, A. C., \& Sanders, J. 2002, MNRAS, 337, 71

Sutherland, R. S., \& Dopita, M. A. 1993, ApJS, 88, 253

Tornatore, L., Borgani, S., Matteucci, F., Recchi, S., \& Tozzi, P. 2004, MNRAS, 349, L19

van de Weygaert, R., \& Bertschinger, E. 1996, MNRAS, 281, 84

van Kampen, E. 1997, in 12th Kingston meeting on Theoretical Astrophysics: Computational Astrophysics, ed. D. A. Clarke, \& M. J. West, ASP Conf. Ser., 123, 231

van Kampen, E., Jimenez, R., \& Peacock, J. A. 1999, MNRAS, 310, 43

Venturi, T., Bardelli, S., Zambelli, G., Morganti, R., \& Hunstead, R. W. 2001, MNRAS, 324, 1131 